MATHEMATICS OF COMPUTATION

Volume 72, Number 243, Pages 1531-1548

S $0025-5718(03) 01474-1$

Article electronically published on January 13, 2003

\title{
COMPUTING THE MULTIPLICATIVE GROUP OF RESIDUE CLASS RINGS
}

\author{
FLORIAN HESS, SEBASTIAN PAULI, AND MICHAEL E. POHST
}

\begin{abstract}
Let $\mathbf{k}$ be a global field with maximal order $\mathfrak{o}_{\mathbf{k}}$ and let $\mathfrak{m}_{0}$ be an ideal of $\mathfrak{o}_{\mathbf{k}}$. We present algorithms for the computation of the multiplicative group $\left(\mathfrak{o}_{\mathbf{k}} / \mathfrak{m}_{0}\right)^{*}$ of the residue class ring $\mathfrak{o}_{\mathbf{k}} / \mathfrak{m}_{0}$ and the discrete logarithm therein based on the explicit representation of the group of principal units. We show how these algorithms can be combined with other methods in order to obtain more efficient algorithms. They are applied to the computation of the ray class group $\mathbf{C l}_{\mathbf{k}}^{\mathfrak{m}}$ modulo $\mathfrak{m}=\mathfrak{m}_{0} \mathfrak{m}_{\infty}$, where $\mathfrak{m}_{\infty}$ denotes a formal product of real infinite places, and also to the computation of conductors of ideal class groups and of discriminants and genera of class fields.
\end{abstract}

\section{INTRODUCTION}

Let $\mathbf{k}$ be a number field with ring of integers $\emptyset_{\mathbf{k}}$ and let $\mathfrak{m}_{0}$ be an ideal of $\varnothing_{\mathbf{k}}$. We describe how a basis of the multiplicative group $\left(\varnothing_{\mathbf{k}} / \mathfrak{m}_{0}\right)^{*}$ can be computed and how the discrete logarithm problem in $\left(\varnothing_{\mathbf{k}} / \mathfrak{m}_{0}\right)^{*}$ can be solved.

Let $\prod_{\mathfrak{p} \mid \mathfrak{m}_{0}} \mathfrak{p}^{m_{\mathfrak{p}}}$ be the decomposition of $\mathfrak{m}_{0}$ into a product of prime ideals. Then the unit group $\left(\varnothing_{\mathbf{k}} / \mathfrak{m}_{0}\right)^{*}$ of the residue class ring $\varnothing_{\mathbf{k}} / \mathfrak{m}_{0}$ satisfies

$$
\left(\varnothing_{\mathbf{k}} / \mathfrak{m}_{0}\right)^{*} \cong \prod_{\mathfrak{p} \mid \mathfrak{m}_{0}}\left(\varnothing_{\mathbf{k}} / \mathfrak{p}^{m_{\mathfrak{p}}}\right)^{*}
$$

Hence, the computation of $\left(\varnothing_{\mathbf{k}} / \mathfrak{m}_{0}\right)^{*}$ is reduced to the computation of all $\left(\varnothing_{\mathbf{k}} / \mathfrak{p}^{m}\right)^{*}$. For nonzero prime ideals $\mathfrak{p}$ of $\varnothing_{\mathbf{k}}$ the completion of $\mathbf{k}$ with respect to the corresponding non-archimedian exponential valuation $v_{\mathfrak{p}}$ is denoted by $\mathbf{k}_{\mathfrak{p}}$. Let $\varnothing_{\mathfrak{p}}$ denote the valuation ring of $\mathbf{k}_{\mathfrak{p}}$ with unique maximal ideal $\mathfrak{b}_{\mathfrak{p}}$. Then

$$
\begin{aligned}
\left(\varnothing_{\mathbf{k}} / \mathfrak{p}^{m}\right)^{*} \cong\left(\varnothing_{\mathfrak{p}} / \mathfrak{b}_{\mathfrak{p}}{ }^{m}\right)^{*} & \cong\left(\varnothing_{\mathfrak{p}} / \mathfrak{b}_{\mathfrak{p}}\right)^{*} \times\left(1+\mathfrak{b}_{\mathfrak{p}}\right) /\left(1+\mathfrak{b}_{\mathfrak{p}}{ }^{m}\right) \\
& \cong\left(\varnothing_{\mathbf{k}} / \mathfrak{p}\right)^{*} \times(1+\mathfrak{p}) /\left(1+\mathfrak{p}^{m}\right)
\end{aligned}
$$

In order to determine $\left(\varnothing_{\mathbf{k}} / \mathfrak{p}^{m}\right)^{*}$ we therefore compute $(\varnothing / \mathfrak{p})^{*}$ and $(1+\mathfrak{p}) /\left(1+\mathfrak{p}^{m}\right)$. Algorithms for the computation of a primitive element of the residue class field $\left(\varnothing_{\mathbf{k}} / \mathfrak{p}\right)^{*}$ are contained in the literature, for instance a method by Gauss in [PZ89]. For a survey of algorithms for the discrete logarithm in the finite field $\left(\varnothing_{\mathbf{k}} / \mathfrak{p}\right)^{*}$ we refer the reader to SWD.

In Section 3 we present a method for the computation of a basis of $(1+\mathfrak{p}) /\left(1+\mathfrak{p}^{m}\right)$ [Pau96] which is derived from the explicit representation of the principal units (or one-units) of a local field as described in Has80 chapter 15].

Received by the editor February 2, 1999 and, in revised form, November 8, 2001.

2000 Mathematics Subject Classification. Primary 11R29, 11R37, 11Y16, 11Y40. 
In Section 4 we present an algorithm for the computation of $(1+\mathfrak{p}) /\left(1+\mathfrak{p}^{m}\right)$ by H. Cohen, M. Olivier and F. Diaz y Diaz [CDO96, CDO98, Coh00] that exploits the isomorphism

$$
\left(1+\mathfrak{p}^{k}\right) /\left(1+\mathfrak{p}^{l}\right) \cong\left(\mathfrak{p}^{k} / \mathfrak{p}^{l}\right)^{+}, \text {for } k \text { subject to } k \leq l \leq 2 k .
$$

They compute successively generators and relations for the groups $\left(1+\mathfrak{p}^{k_{i}}\right) /\left(1+\mathfrak{p}^{l_{i}}\right)$ with $k_{i}=2^{i}<m, l_{i}=\min \left(2^{i+1}, m\right)$, and combine these.

In Section 5 we describe how the $\mathfrak{p}$-adic logarithm and the Artin-Hasse logarithm can be used to solve the discrete logarithm problem. This is described in detail in [Coh00, chapter 4].

In Section [ we present an efficient algorithm for the computation of a basis of the group of principal units and for the discrete logarithm combining methods from Sections [3, 4 and 5.

Applications of these algorithms to the computation of ray class groups, their conductors, and the discriminants and signatures of the respective ray class fields are presented in Sections 7 and 8 . They are also an important tool in the computation of ray class fields Fie00, Coh00.

In Section 9 we describe the algorithms in the case where $\mathbf{k}$ is a global function field.

\section{Notation}

Throughout the paper a finite abelian group $G$ is presented by a column vector $g \in G^{m}$, whose entries form a system of generators for $G$, and by a matrix of relations $M \in \mathbb{Z}^{m \times n}$ of rank $m$, such that $v^{T} g=0$ for $v \in \mathbb{Z}^{m}$ if and only if $v$ is an integral linear combination of the rows of $M$. We note that for every $a \in G$ there is a $v \in \mathbb{Z}^{m}$ satisfying $a=v^{\mathrm{T}} g$. If $g_{1}, \ldots, g_{m}$ is a basis of $G, M$ is usually a diagonal matrix. Algorithms for calculations with finite abelian groups can be found in [Coh00] and Sim94. If $G$ is a multiplicative abelian group, then $v^{\mathrm{T}} g$ is an abbreviation for $g_{1}^{v_{1}} \cdots g_{m}^{v_{m}}$.

We denote the degree of $\mathbf{k}$ over $\mathbb{Q}$ by $n$. We denote an integral basis of the ring of integers $\emptyset_{\mathbf{k}}$ of $\mathbf{k}$ by a vector $w \in \emptyset_{\mathbf{k}}{ }^{n}$. A matrix representation of an ideal $\mathfrak{a} \subset \varnothing_{\mathbf{k}}$ is a matrix $A \in \mathbb{Z}^{n \times n}$ such that $A w$ is a $\mathbb{Z}$ basis of $\mathfrak{a}$. If $\mathfrak{p} \subset \emptyset_{\mathbf{k}}$ is a prime ideal, we write $e$ for the ramification index and $f$ for the inertia degree of $\mathfrak{p}$.

For the complexity considerations we fix the following notation. Let $R$ be a ring and $\mathfrak{a}$ an ideal of $R$.

- We denote by $\mathrm{M}_{\mathfrak{a}}$ the number of bit operations needed for multiplying two elements in $R$ modulo a.

$\circ$ Let $a, b \in R$. The number of bit operations needed for finding an element $q \in R$ with $a \equiv q \cdot b \bmod \mathfrak{a}$ is denoted by $\mathrm{D}_{\mathfrak{a}}$.

- The number of bit operations for multiplying a $k \times l$ matrix by an $l \times m$ matrix over a ring $R$ is denoted by $\mathrm{M}_{R}(k, l, m)$.

- Denote by $\mathrm{T}_{R}(n)$ the number of bit operations required for triangularizing an $n \times n$ matrix over a principal ideal ring $R$.

- Let $A$ be a matrix in $\mathbb{Z}^{n \times n}$ whose coefficients are bounded by $a$. We denote by $\mathrm{S}_{a}$ the number of bit operations needed to compute the Smith Normal Form $S$ of $A$ and transformation matrices $T_{L}$ and $T_{R}$ such that $S=T_{L} A T_{R}$. 


\section{PRINCIPAL UNITS}

In this section we present several results about principal units (also called oneunits) (for details we refer to [Has80, chapter 15]), and apply these to the computation of the multiplicative group of residue class rings Pau96. We assume that $p$ is the unique rational prime contained in $\mathfrak{p}$. An element $\eta_{\nu} \in 1+\mathfrak{b}_{\mathfrak{p}}$ is called a principal unit of level $\nu$, iff $\eta_{\nu} \equiv 1 \bmod \mathfrak{b}_{\mathfrak{p}}{ }^{\nu}$. Every principal unit $\eta \in 1+\mathfrak{b}_{\mathfrak{p}}$ has a unique representation $\eta=\prod_{\nu=1}^{\infty}\left(1+a_{\nu} \pi^{\nu}\right)$, where the $a_{\nu}$ are from a fixed set of representatives of $\varnothing_{\mathfrak{p}} / \mathfrak{b}_{\mathfrak{p}}$ and $\pi \in \mathfrak{p} \backslash \mathfrak{p}^{2}$. The groups $\left(1+\mathfrak{b}_{\mathfrak{p}}{ }^{m}\right) /\left(1+\mathfrak{b}_{\mathfrak{p}}{ }^{m+1}\right)$ are isomorphic to $\left(\varnothing_{\mathfrak{p}} / \mathfrak{b}_{\mathfrak{p}}\right)^{+}$.

The $p$-th power rule yields that the $p$-th powers of principal units generators of a level are generators for principal units of further levels.

Theorem 3.1 ( $p$-th power rule). Let $e$ be the ramification index of $\mathfrak{b}_{\mathfrak{p}}$ and $\eta_{\nu} \equiv$ $1+a \pi^{\nu} \bmod \mathfrak{b}_{\mathfrak{p}}{ }^{\nu+1}$, where $a$ is in $\emptyset_{\mathfrak{p}}$. Let $p=-\pi^{e} \varepsilon$ be the factorisation of $p$, where $\varepsilon$ is a unit. Then the $p$-th power of $\eta_{\nu}$ satisfies

$$
\eta_{\nu}^{p} \equiv\left\{\begin{array}{lllll}
1 & +a^{p} \pi^{p \nu} & \bmod ^{*} & \mathfrak{b}_{\mathfrak{p}}{ }^{p \nu+1} & \text { if } \quad \nu<\frac{e}{p-1} \\
1+ & \left(a^{p}-\varepsilon a\right) \pi^{p \nu} & \bmod ^{*} & \mathfrak{b}_{\mathfrak{p}}{ }^{p \nu+1} & \text { if } \nu=\frac{e}{p-1} \\
1-\varepsilon a \pi^{\nu+e} & \bmod ^{*} & \mathfrak{b}_{\mathfrak{p}}{ }^{\nu+e+1} & \text { if } \nu>\frac{e}{p-1}
\end{array}\right.
$$

The maps $h_{1}: a+\mathfrak{b}_{\mathfrak{p}} \longmapsto a^{p}+\mathfrak{b}_{\mathfrak{p}}$ and $h_{3}: a+\mathfrak{b}_{\mathfrak{p}} \longmapsto-\varepsilon a+\mathfrak{b}_{\mathfrak{p}}$ are automorphisms of $\left(\varnothing_{\mathfrak{p}} / \mathfrak{b}_{\mathfrak{p}}\right)^{+}$, whereas $h_{2}: a+\mathfrak{b}_{\mathfrak{p}} \longmapsto a^{p}-\varepsilon a+\mathfrak{b}_{\mathfrak{p}}$ is in general only a homomorphism.

This has the following consequence. If $\eta_{1 \nu}, \ldots, \eta_{f \nu}$ is a system of generators for the level $\nu<\frac{e}{p-1}$ (for the level $\nu>\frac{e}{p-1}$ ), then $\eta_{1 \nu}^{p}, \ldots, \eta_{f \nu}^{p}$ is a system of generators for the level $p \nu$ (for the level $\nu+e$ ). Levels based on the level $\nu=\frac{e}{p-1}$ need to be discussed separately.

Lemma 3.2. The kernel of $h_{2}$ is of order 1 or $p$.

The systems of generators for the levels $\nu \geq \frac{e}{p-1}+e=\frac{p e}{p-1}$ are obtained from the systems of generators for the levels $\nu^{\prime}<\frac{p e}{p-1}$. The systems of generators for the levels $\nu$ with $p \mid \nu$ are obtained from systems of generators for lower levels. We define the set of fundamental levels $F_{e}$ of $\mathfrak{b}_{\mathfrak{p}}$ by

$$
F_{e}:=\left\{\nu \mid 0<\nu<\frac{p e}{p-1}, p \nmid \nu\right\} .
$$

All levels can be obtained from the fundamental levels via the substitutions presented above. Note that the cardinality of $F_{e}$ is $e$.

The next statement is deduced from the basis representation of the principal units in Hasse's book [Has80, p. 238]. The proof also gives an algorithm for solving the discrete logarithm problem in $(1+\mathfrak{p}) /\left(1+\mathfrak{p}^{m}\right)$.

Theorem 3.3 (Basis representation, case I). If

i) $(p-1)$ does not divide $e$, or

ii) $h_{2}$ is an isomorphism, or

iii) $m<\frac{e}{p-1}$,

then the class $[\eta] \in\left(1+\mathfrak{b}_{\mathfrak{p}}\right) /\left(1+\mathfrak{b}_{\mathfrak{p}}{ }^{m}\right)$ has the basis representation

$$
[\eta]=\prod_{\nu \in F_{e}} \prod_{i=1}^{f}\left[\eta_{i \nu}\right]^{a_{i \nu}} \quad\left(0 \leq a_{i \nu}<\operatorname{ord}\left(\left[\eta_{i \nu}\right]\right)\right)
$$


The $\eta_{i \nu}$ are given in the form $\eta_{i \nu}=1+\omega_{i} \pi^{\nu}$ for a fixed set of representatives of a $\mathbb{Z} / p \mathbb{Z}$-basis $\omega_{1}, \ldots, \omega_{f}$ of $\emptyset_{\mathfrak{p}} / \mathfrak{b}_{\mathfrak{p}}$ in $\emptyset_{\mathfrak{p}}$. The order of $\eta_{i \nu}$ is $\operatorname{ord}\left(\left[\eta_{i \nu}\right]\right)=p^{s_{\nu}}$, with

$$
\begin{aligned}
& s_{\nu}= \begin{cases}0, & \nu \geq m, \\
s_{1 \nu}+s_{2 \nu}+1, & \nu<m,\end{cases} \\
& s_{1 \nu}= \begin{cases}\left\lfloor\log _{p} \frac{m-1}{\nu}\right\rfloor, & m \leq \frac{p e}{p-1}, \\
\left.\log _{p} \frac{p e}{\nu(p-1)}\right\rfloor, & m>\frac{p e}{p-1},\end{cases} \\
& s_{2 \nu}= \begin{cases}0, & m \leq \frac{p e}{p-1}, \\
\left\lfloor\frac{m-1-\nu^{s_{1 \nu}}}{e}\right\rfloor, & m>\frac{p e}{p-1} .\end{cases}
\end{aligned}
$$

Proof. We begin by computing the orders of the $\eta_{i \nu}$, and show that we have a basis representation afterwards.

If $\nu \geq m$, then $\operatorname{ord}\left(\left[\eta_{i \nu}\right]\right)=1$. For $\nu<m \leq \frac{p e}{p-1}$ the generators of the fundamental level $\nu \in F_{e}$ are generators of the levels $p^{s_{1}} \nu$, where $s_{1} \in \mathbb{Z}^{>0}$ with $p^{s_{1}} \nu<m$.

We now look for the maximal $s_{1 \nu}$ fulfilling the last inequality. With $p^{s_{1 \nu}} \nu \leq m-1$ we get $s_{1 \nu}=\left\lfloor\log _{p}\left(\frac{m-1}{\nu}\right)\right\rfloor$. Hence $p^{s_{1 \nu}} \nu$ is the maximal level $\left(1+\mathfrak{b}_{\mathfrak{p}}\right) /\left(1+\mathfrak{b}_{\mathfrak{p}}{ }^{m}\right)$ for which $\eta_{i \nu}$ is a system of generators. With the $p$-th power rule and the choice of $s_{1 \nu}$ we get $\eta_{i \nu}^{p^{s_{1 \nu}+1}} \equiv 1 \bmod \mathfrak{b}_{\mathfrak{p}}{ }^{m}$. Hence the order of the class $\left[\eta_{i \nu}\right]$ in $\left(1+\mathfrak{b}_{\mathfrak{p}}\right) /\left(1+\mathfrak{b}_{\mathfrak{p}}{ }^{m}\right)$ is $p^{s_{1 \nu}+1}$.

For $m>\frac{p e}{p-1}$ the $\eta_{i \nu}$ are the generators of the levels $p^{s_{1}} \nu+s_{2} e$, where $s_{1}, s_{2} \in \mathbb{Z}^{>0}$ satisfy $p^{s_{1}} \nu+s_{2} e<m$ and $p^{s_{1}} \nu<\frac{p e}{p-1}$. We are looking for the maximal level whose generators are $p$-th powers of $\eta_{i \nu}$. As above, $s_{1 \nu}$ is calculated for $m=\frac{p e}{p-1}$. Then $p^{s_{1 \nu}} \nu+s_{2} e \leq m-1$, and we get $s_{2 \nu}=\left\lfloor\frac{m-1-p^{s_{1 \nu}}}{e}\right\rfloor$ for the maximal $s_{2 \nu}$ for which the inequality holds. As above, the order of $\left[\eta_{i \nu}\right]$ is increased by a power of $p$, and we obtain $s_{\nu}=s_{1 \nu}+s_{2 \nu}+1$.

We show by induction on $m$ that we indeed have a basis representation. Let $\omega_{1}, \ldots, \omega_{f}$ be fixed representatives of a $\mathbb{Z} / p \mathbb{Z}$-basis of $\emptyset_{\mathfrak{p}} / \mathfrak{b}_{\mathfrak{p}}$ in $\varnothing_{\mathfrak{p}}$, and $\eta \in 1+\mathfrak{b}_{\mathfrak{p}}$. We denote the class of $\eta$ in $\left(1+\mathfrak{b}_{\mathfrak{p}}\right) /\left(1+\mathfrak{b}_{\mathfrak{p}}{ }^{k}\right)$ by $[\eta]_{k}$.

We start with the case $(p-1) \nmid e$. The class of $\eta$ in $\left(1+\mathfrak{b}_{\mathfrak{p}}\right) /\left(1+\mathfrak{b}_{\mathfrak{p}}{ }^{2}\right)$ has the basis representation

$$
\begin{gathered}
\eta \equiv \prod_{\nu \in F_{e}} \prod_{i=1}^{f}\left(1+\omega_{i} \pi^{\nu}\right)^{a_{i \nu}} \bmod \mathfrak{b}_{\mathfrak{p}}{ }^{2} \\
\left(0 \leq a_{i \nu}<\operatorname{ord}\left(\left[1+\omega_{i} \pi^{\nu}\right]_{1}\right)=\left\{\begin{array}{cc}
1, & \nu \neq 1 \\
p, & \nu=1
\end{array}\right) .\right.
\end{gathered}
$$

Next we assume that the basis representation of $[\eta]_{k}$ in $\left(1+\mathfrak{b}_{\mathfrak{p}}\right) /\left(1+\mathfrak{b}_{\mathfrak{p}}{ }^{k}\right)$ is known for $k<m$ :

$$
\eta \equiv \prod_{\nu \in F_{e}} \prod_{i=1}^{f}\left(1+\omega_{i} \pi^{\nu}\right)^{a_{i \nu}} \bmod \mathfrak{b}_{\mathfrak{p}}{ }^{k} \quad\left(0 \leq a_{i \nu}<\operatorname{ord}\left(\left[1+\omega_{i} \pi^{\nu}\right]_{k}\right)\right) .
$$

We construct $b_{11}, \ldots, b_{f e}$ satisfying

$$
\eta \equiv \prod_{\nu \in F_{e}} \prod_{i=1}^{f}\left(1+\omega_{i} \pi^{\nu}\right)^{b_{i \nu}} \bmod \mathfrak{b}_{\mathfrak{p}}{ }^{k+1} \quad\left(0 \leq b_{i \nu}<\operatorname{ord}\left(\left[1+\omega_{i} \pi^{\nu}\right]_{k+1}\right)\right) .
$$


Let $\nu^{\prime} \in F_{e}$ be the fundamental level for $k$. There exist $s_{1}, s_{3}$ with $s_{1}=$ $\max \left\{s \in \mathbb{Z} \mid \nu^{\prime} p^{s}<\min \left(k, \frac{p e}{p-1}\right)\right\}$ and $k=\nu^{\prime} p^{s_{1}}+s_{3} e$. Then

$$
\eta^{\prime}=\frac{\eta}{\prod_{\nu \in F_{e}} \prod_{i=1}^{f}\left(1+\omega_{i} \pi^{\nu}\right)^{a_{i \nu}}}
$$

is a principal unit of level $k$. Let $\omega_{1}^{\prime}, \ldots, \omega_{f}^{\prime}$ be subject to $\omega_{i}^{\prime}=h_{3}^{s_{3}}\left(h_{1}^{s_{1}}\left(\omega_{i}\right)\right)$. There exist $c_{1}, \ldots, c_{f}$ with $0 \leq c_{i}<p$ and

$$
\eta^{\prime} \equiv \prod_{i=1}^{f}\left(1+\omega_{i}^{\prime} \pi^{k}\right)^{c_{i}} \bmod \mathfrak{b}_{\mathfrak{p}}{ }^{k+1}
$$

Hence, we obtain

$$
\eta \equiv \prod_{\nu \in F_{e}} \prod_{i=1}^{f}\left(1+\omega_{i} \pi^{\nu}\right)^{a_{i \nu}} \prod_{i=1}^{f}\left(1+\omega_{i}^{\prime} \pi^{k}\right)^{c_{i}} \bmod \mathfrak{b}_{\mathfrak{p}}{ }^{k+1} .
$$

The $\omega_{i}$ were chosen to satisfy $\left(1+\omega_{i} \pi^{\nu^{\prime}}\right)^{p^{s_{1}+s_{2}}} \equiv\left(1+\omega_{i}^{\prime} \pi^{k}\right) \bmod \mathfrak{b}_{\mathfrak{p}}{ }^{k+1}$, and we conclude that

$$
\prod_{i=1}^{f}\left(1+\omega_{i}^{\prime} \pi^{k}\right)^{c_{i}} \equiv \prod_{i=1}^{f}\left(1+\omega_{i}^{\prime} \pi^{\nu^{\prime}}\right)^{c_{i} p^{s_{1}+s_{2}}} \bmod \mathfrak{b}_{\mathfrak{p}}{ }^{k+1} .
$$

With $b_{i \nu}:=\left\{\begin{array}{ll}a_{i \nu}, & \nu \neq \nu^{\prime}, \\ a_{i \nu}+c_{i} p^{s_{1}+s_{2}}, & \nu=\nu^{\prime},\end{array}\right.$ we finally get the representation

$$
\begin{gathered}
\eta \equiv \prod_{\nu \in F_{e}} \prod_{i=1}^{f}\left(1+\omega_{i} \pi^{\nu}\right)^{b_{i \nu}} \bmod \mathfrak{b}_{\mathfrak{p}}{ }^{k+1} \\
\left(0 \leq b_{i \nu}<\left\{\begin{array}{ll}
\operatorname{ord}\left(\left[1+\omega_{i} \pi^{\nu}\right]_{k}\right), & \nu \neq \nu^{\prime} \\
p \operatorname{ord}\left(\left[1+\omega_{i} \pi^{\nu}\right]_{k}\right), & \nu=\nu^{\prime}
\end{array}\right) .\right.
\end{gathered}
$$

The generators of the level $\nu^{\prime}$ are the generators of the new level $k$. It follows from the $p$-th power rule that the order of $\left(1+\omega_{i} \pi^{\nu^{\prime}}\right)$ increases by the factor $p$ and that the orders of the other generators do not change.

In case $(p-1) \mid e$ the proof is analogous; we just need to use a different isomorphism, $h_{2}: \omega \mapsto p \omega-\varepsilon \omega$, to proceed from level $\frac{e}{p-1}$ to level $\frac{p e}{p-1}$.

Proposition 3.4. In case I (see Theorem 3.3) a basis of $(1+\mathfrak{p}) /(1+\mathfrak{p})^{m}$ can be computed in $O\left(\right.$ ef $\left.\log \frac{p e}{p-1} \mathbf{M}_{\mathfrak{p}^{m}}\right)$ bit operations.

The multiplicative groups $\left(1+\mathfrak{b}_{\mathfrak{p}}\right) /\left(1+\mathfrak{b}_{\mathfrak{p}}{ }^{m}\right)$ and $(1+\mathfrak{p}) /\left(1+\mathfrak{p}^{m}\right)$ are isomorphic, and since we chose $\pi$ and $\omega_{1}, \ldots, \omega_{f}$ in $\emptyset_{\mathbf{k}}$, the generators of $\left(1+\mathfrak{b}_{\mathfrak{p}}\right) /\left(1+\mathfrak{b}_{\mathfrak{p}}{ }^{m}\right)$ are generators of $(1+\mathfrak{p}) /\left(1+\mathfrak{p}^{m}\right)$.

Hence, a basis of the group $(1+\mathfrak{p}) /\left(1+\mathfrak{p}^{m}\right)$ can be computed easily if $(p-1)$ does not divide $e$ or $h_{2}$ is an isomorphism or $m<\frac{e}{p-1}$. The proof of Theorem 3.3 above yields an algorithm for solving the discrete logarithm problem in $(1+\mathfrak{p}) /\left(1+\mathfrak{p}^{m}\right)$.

Algorithm 3.5 (Discrete logarithm, principal units, case I).

Input: $\quad \eta \in 1+\mathfrak{p}, \emptyset_{\mathbf{k}}, \mathfrak{p} \subset \emptyset_{\mathbf{k}}, e, f, p, m \in \mathbb{Z}^{>0}$, a basis $\left(\eta_{11}, \ldots, \eta_{f e}\right)$ of

$(1+\mathfrak{p}) /\left(1+\mathfrak{p}^{m}\right)$ and a system of representatives $\left(\omega_{1}, \cdots, \omega_{n}\right)$ of a $\mathbb{Z} / p \mathbb{Z}$-basis of $\varnothing_{\mathbf{k}} / \mathfrak{p}$ in $\varnothing_{\mathbf{k}}$ as in Theorem 3.3.

Output: $a \in \mathbb{Z}^{e f}$ such that $\left[a^{T}\left(\eta_{11}, \ldots, \eta_{f e}\right)\right]=[\eta]$. 
○ Set $a \leftarrow 0 \in \mathbb{Z}^{\text {fe }}$.

○ For all levels $1 \leq \nu<m$ do:

- Find base level $\nu^{\prime}$ of $\nu$.

- Compute the number of substitutions $s_{1}, s_{2}, s_{3}$.

○ Set $\omega_{i}^{\prime} \leftarrow h_{3}^{s_{3}}\left(h_{2}^{s_{2}}\left(h_{1}^{s_{1}}\left(\omega_{i}\right)\right)\right)$ for $i=1, \ldots, f$.

- Find $c_{1}, \ldots, c_{f}$, such that $(\eta-1) / \pi^{\nu} \equiv \sum_{i=1}^{f} c_{i} \omega_{i}^{\prime} \bmod \mathfrak{p}$.

○ Replace $a_{1 \nu^{\prime}} \leftarrow a_{1 \nu^{\prime}}+p^{s_{1}+s_{2}+s_{3}} c_{1}, \ldots, a_{f \nu^{\prime}} \leftarrow a_{f \nu^{\prime}}+p^{s_{1}+s_{2}+s_{3}} c_{f}$.

○ Replace $\eta \leftarrow \eta / \prod_{i=1}^{f} \eta_{i \nu^{\prime}}^{p^{s_{1}+s_{2}+s_{3}} c_{i}}$.

○ Return $a$.

For each computation of the discrete logarithm with Algorithm 3.5 the values $h_{3}^{s_{3}}\left(h_{2}^{s_{2}}\left(h_{1}^{s_{1}}\left(\omega_{i}\right)\right)\right)$ and $\eta_{i \nu}^{p^{s_{1}+s_{2}+s_{3}}}$ have to be computed. This can be done in $O\left(m e f \log p \mathrm{M}_{\mathfrak{p}^{m}}\right)$ and $O\left(m \mathrm{M}_{\mathfrak{p}^{m}}\right)$ bit operations respectively.

Assuming the data above is known, Algorithm 3.5 returns the discrete logarithm of $\eta \in(1+\mathfrak{p}) /(1+\mathfrak{p})^{m}$ in $O\left(m\left(\mathrm{D}_{\phi_{\mathbf{k}}}^{\mathfrak{p}^{m}}+\mathrm{T}_{\mathbb{F}_{p}}(f)+f \log p \cdot \mathrm{M}_{\mathfrak{p}^{m}}\right)\right)$ bit operations.

If $(p-1)$ divides $e$ and $h_{2}$ is not an isomorphism, the generators of the level $\frac{e}{p-1}$ are not a complete system of generators for the level $\frac{p e}{p-1}$. In order to obtain the generators of the level $e /(p-1)$, the substitution $h_{1}$ is applied $p^{\mu_{0}-1}$ times to the generators of the fundamental step $e_{0}$, where $p^{\mu_{0}-1}(p-1) e_{0}=e$ and $p$ does not divide $e_{0}$. Since the order of the kernel of $h_{2}$ is $p$ in this case, we can find a $\mathbb{Z} / p \mathbb{Z}$-basis $\omega_{1}, \ldots, \omega_{f}$ with $\omega_{1}^{p^{\mu_{0}}}-\varepsilon \omega_{1}^{p^{\mu_{0}-1}} \equiv 0 \bmod \mathfrak{p}$, so that $\omega_{1}^{p^{\mu_{0}}}$ generates the kernel of $h_{2}$. To complete the system of generators for the level $\frac{p e}{p-1}$, we introduce an additional generator $\omega_{*}$ of $\varnothing_{\mathbf{k}} / \mathfrak{p}$ which is not contained in the image of $h_{2}$. If $x^{p}-\varepsilon x \equiv \omega_{*} \bmod \mathfrak{p}$ has no solution, then $\omega_{*}$ is not contained in the image of $h_{2}$.

Theorem 3.6 (Unique representation, case II). Let $(p-1) \mid e$, and let $h_{2}$ not be an isomorphism. Choose $e_{0}$ and $\mu_{0}$ such that $e=p^{\mu_{0}-1}(p-1) e_{0}$ and $p$ does not divide $e_{0}$. Let $\omega_{1}, \ldots, \omega_{f}$ be a $\mathbb{Z} / p \mathbb{Z}$-basis of $\emptyset_{\mathbf{k}} / \mathfrak{p}$ in $\emptyset_{\mathbf{k}}$ subject to $\omega_{1}^{p^{\mu_{0}}}-\varepsilon \omega_{1}^{p^{\mu_{0}-1}} \equiv$ $0 \bmod \mathfrak{p}$. Choose $\omega_{*} \in \varnothing_{\mathbf{k}}$ such that $x^{p}-\varepsilon x \equiv \omega_{*} \bmod \mathfrak{p}$ has no solution. Then the class $[\eta] \in(1+\mathfrak{p}) /\left(1+\mathfrak{p}^{m}\right)$ has the unique representation

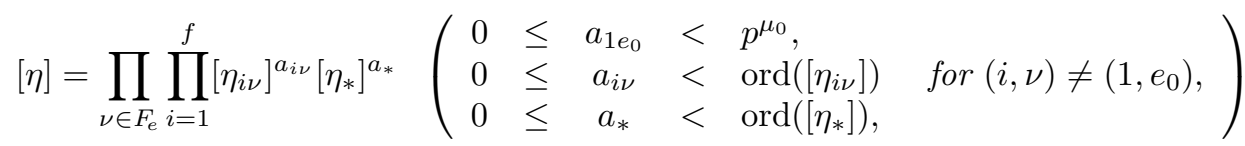

with $\eta_{i \nu}=1+\omega_{i} \pi^{\nu}$ and $\eta_{*}=1+\omega_{*} \pi^{p^{\mu_{0}} e_{n} u l}$. The corresponding orders are $\operatorname{ord}\left(\left[\eta_{i \nu}\right]\right)=p^{s_{\nu}}$ for $(i, \nu) \neq\left(1, e_{0}\right)$ and $\operatorname{ord}\left(\left[\eta_{*}\right]\right)=p^{s_{*}+1}$, respectively, where $s_{*}=\left\lfloor\frac{m-1-p^{\mu_{0}} e_{0}}{e}\right\rfloor$ and $s_{\nu}$ is as defined in Theorem 3.3 .

Proof. By the choice of $\omega_{1}$ and $\omega_{*}$, the representation is unique. The order of $\left[\eta_{*}\right]$ is obtained as follows. $e_{0}$ and $\mu_{0}$ were chosen subject to $\frac{p e}{p-1}=p^{\mu_{0}} e_{0}$; therefore $\eta_{*}=1+\omega_{0} \pi^{p^{\mu_{0}}} e_{0}$ is a principal unit of level $p^{\mu_{0}} e_{0}$. The $p$-th powers of $\left[\eta_{*}\right]$ are contained in the systems of generators of the levels $p^{\mu_{0}} e_{0}+s e$. The maximal nonnegative $s$ satisfying $p^{\mu_{0}} e_{0}+s e \leq m-1$ is $s_{*}=\left\lfloor\frac{m-1-p^{\mu_{0}} e_{0}}{e}\right\rfloor$.

We remark that we do not necessarily get a basis representation, since the order of $\eta_{1 e_{0}}$ is in general not $p^{\mu_{0}}$. However, in many cases the unique representation is a basis representation.

Corollary 3.7 (Basis representation, case IIa). If $(p-1) \mid e$ and the kernel of $h_{2}$ is not trivial and 
i) $p \leq 3$ and $m \leq 2 e$ or

ii) $p>3$ and $(p-1) m \leq(p+1) e$,

then the unique representation in Theorem 3.6 is a basis representation. In that case we have ord $\left(\left[\eta_{1 e_{0}}\right]\right)=p^{\mu_{0}}$.

Proof. Let $\nu=\frac{e}{p-1}=p^{\mu_{0}-1} e_{0}$ and $\eta=\eta_{1 e_{0}}^{p^{\mu_{0}-1}}=\left(1+\omega_{1} \pi^{e_{0}}\right)^{p^{\mu_{0}-1}}$; then $\eta \equiv$ $1+a \pi^{\nu} \bmod \mathfrak{p}^{\nu+1}$. We determine the maximal exponent $m$ for which the congruence $\eta^{p} \equiv 1 \bmod \mathfrak{p}^{m}$. With the $p$-th power rule and the choice of $\omega_{1}$ we get $\eta^{p} \equiv$ $1 \bmod \mathfrak{p}^{p \nu+1}$. Comparing the summands with larger powers of $p$, the proof of the $p$-th power rule in [Has80, p. 229] shows that

$$
m \leq \min (2 \nu+e,(p-1) \nu+e) .
$$

In the unique representation of $\eta_{1 e_{0}}$ the exponent $a_{1 e_{0}}$ was bounded by $a_{1 e_{0}}<$ $p^{\mu_{0}}$. We can represent $\eta_{1 e_{0}}^{p^{\mu_{0}}}$ by the other generators and get a relation $\left[\eta_{1 e_{0}}^{p^{\mu_{0}}}\right]=$

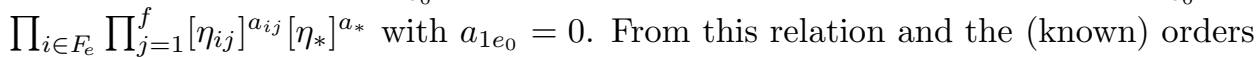
of the other generators a basis of the group $(1+\mathfrak{p}) /(1+\mathfrak{p})^{m}$ can be derived by computing the Smith Normal Form of the relation matrix.

A unique representation of an element in $(1+\mathfrak{p}) /(1+\mathfrak{p})^{m}$ can be obtained easily with a slightly modified version of Algorithm 3.5. If the base level $\nu^{\prime}$ of a level $\nu$ is $e_{0}$ and $\nu \geq p e /(p-1)$, then $\omega_{1}^{\prime}$ is set equal to $h_{3}^{s_{3}}\left(\omega_{*}\right)$ and the vector $a$ representing $\eta$ up to level $\nu$ has to be altered accordingly (note that $a \in \mathbb{Z}^{f e+1}$ in this case).

To obtain a set of generators of $(1+\mathfrak{p}) /\left(1+\mathfrak{p}^{m}\right)$ in case II and case IIa, the elements $\omega_{1}$ and $\omega_{*}$ must be computed. Denote by $Q$ the matrix representing the Frobenius automorphism $x \mapsto x^{p}$ in the finite field $\varnothing_{\mathbf{k}} / \mathfrak{p}$. Then $\omega_{0}$ is the $p^{\mu_{0}-1}$-th root of a nontrivial element from the kernel of $Q-\varepsilon I$, and $\omega_{*}$ can be chosen from $\emptyset_{\mathbf{k}} / \mathfrak{p} \backslash \operatorname{range}(Q-\varepsilon I)$.

Proposition 3.8. In case IIa (see Corollary 3.7) a basis of $(1+\mathfrak{p}) /\left(1+\mathfrak{p}^{m}\right)$ can be computed in

$$
O\left(\text { ef } \log \frac{p e}{p-1} \mathbf{M}_{\mathfrak{p}^{m}}+\mathrm{T}_{\mathbb{F}_{p}}(f)\right)
$$

bit operations. In case II (see Theorem [3.6) a set of generators and a relation matrix can be computed in

$$
O\left(e f \log p\left(m+\frac{e}{p-1}\right) \mathbf{M}_{\mathfrak{p} m}+m\left(\mathrm{D}_{\mathfrak{p} m}+\mathrm{T}_{\mathbb{F}_{p}}(f)\right)\right)
$$

bit operations.

Let $\left(\left(\eta_{11}, \ldots, \eta_{f e}, \eta_{*}\right), M\right)$ be a representation of $(1+\mathfrak{p}) /\left(1+p^{m}\right)$ as given by Theorem 3.6. Let $S=T_{L} M T_{R}$ be the Smith normal form of $M$. Then

$$
\left(T_{R}\left(\eta_{11}, \ldots, \eta_{f e}, \eta_{*}\right)^{T}\right)^{T}
$$

is a basis of $(1+\mathfrak{p}) /\left(1+\mathfrak{p}^{m}\right)$ which can be computed in $O\left(\left(e f m \log p+e^{2} f^{2}\right) \mathrm{M}_{\mathfrak{p}}^{m}\right)$ bit operations.

Proposition 3.9. In case II a basis of the group $(1+\mathfrak{p}) /\left(1+\mathfrak{p}^{m}\right)$ can be obtained in

$$
O\left(e f\left(m \log p+\frac{e}{p-1} \log p+e f\right) \mathrm{M}_{\mathfrak{p} m}+m\left(\mathrm{D}_{\mathfrak{p} m}+\mathrm{T}_{\mathbb{F}_{p}}(f)\right)+\mathrm{S}(e f)\right)
$$


bit operations. The discrete logarithm of an element can be computed in

$$
O\left(m\left(\mathrm{D}_{\phi_{\mathbf{k}}}^{\mathfrak{p}^{m}}+\mathrm{T}_{\mathbb{F}_{p}}(f)+f \log p \cdot \mathrm{M}_{\mathfrak{p}^{m}}\right)+\mathrm{M}(1, e f, e f)\right)
$$

bit operations.

\section{Quadratic methods}

The quadratic method of Cohen, Diaz y Diaz, and Olivier CDO96, CD098. Coh00] is based on the following fact.

Lemma 4.1. Let $\mathbf{k}$ be an algebraic number field with maximal order $\varnothing_{\mathbf{k}}$, and let $\mathfrak{p} \subset \varnothing_{\mathbf{k}}$ be a prime ideal. If $l \leq m \leq 2 l$, then

$$
\Psi:\left(1+\mathfrak{p}^{l}\right) /\left(1+\mathfrak{p}^{m}\right) \rightarrow\left(\mathfrak{p}^{l} / \mathfrak{p}^{m}\right)^{+},[1+a] \mapsto \bar{a},
$$

is an isomorphism.

A basis of the additive group $\mathfrak{p}^{l} / \mathfrak{p}^{m}$ can be computed easily. Let $w$ be an integral basis of $\phi_{\mathbf{k}}$, and let $\mathfrak{p}^{l}$ and $\mathfrak{p}^{m}$, where $1 \leq l \leq m$, be given by the matrices $A$ and $B$, so that $w_{l}=A w$ (respectively $w_{m}=B w$ ) is a $\mathbb{Z}$-basis of $\mathfrak{p}^{l}$ (respectively $\left.\mathfrak{p}^{m}\right)$. The matrix $B A^{-1}$ represents the elements of $\mathfrak{p}^{m}$ by the elements of $\mathfrak{p}^{l}$, as $w_{m}=B A^{-1} A w=B A w_{l}$ Thus $B A^{-1}$ is the matrix of relations of the additive group $\mathfrak{p}^{l} / \mathfrak{p}^{m}$, and the components of $w_{l}$ are its generators. Hence $\mathfrak{p}^{l} / \mathfrak{p}^{m}$ is represented by $\left(w_{l}, B A^{-1}\right)$. If $w_{l}=\left(w_{l 1}, \cdots, w_{l n}\right)^{T}$, we get the representation

$$
(g, M):=\left(\left(\begin{array}{c}
1+w_{l 1} \\
\vdots \\
1+w_{l n}
\end{array}\right), B A^{-1}\right)
$$

for $\left(1+\mathfrak{p}^{l}\right) /\left(1+\mathfrak{p}^{m}\right)$ by generators and relations.

In order to obtain a representation of $[b] \in\left(1+\mathfrak{p}^{l}\right) /\left(1+\mathfrak{p}^{m}\right)$ by $g$, we need to find $a=\left(a_{1}, \cdots, a_{n}\right)$ with $[b-1]=\left[\sum_{i=1}^{n} a_{i} w_{l i}\right]$; then $[b]=\left[\prod_{i=1}^{n}\left(1+w_{l} i\right)^{a_{i}}\right]$ by Lemma 4.1 Let $b \in \mathbb{Z}^{n}$ with $b-1=b w$; then $b w=a A w$. A solution $a \in \mathbb{Z}^{n}$ of the equation $b=a A$ gives the desired representation.

Let $k, l, m$ with $k \leq l \leq m$ and $l \leq m \leq 2 l$ be given. Denote the class $1+a$ modulo $\left(1+\mathfrak{p}^{j}\right)$ by $[1+a]_{j}$.

By the isomorphism theorem the sequence

$$
\begin{aligned}
& 1 \rightarrow\left(1+\mathfrak{p}^{l}\right) /\left(1+\mathfrak{p}^{m}\right) \stackrel{\Psi}{\rightarrow}\left(1+\mathfrak{p}^{k}\right) /\left(1+\mathfrak{p}^{m}\right) \stackrel{\Phi}{\rightarrow}\left(1+\mathfrak{p}^{k}\right) /\left(1+\mathfrak{p}^{l}\right) \rightarrow 1, \\
& {[1+a]_{m} \quad \mapsto \quad[1+a]_{m} \quad \mapsto \quad[1+a]_{l},}
\end{aligned}
$$

is exact.

By the definition of the map $\Phi$ we have $\Phi\left([h]_{m}\right)=[h]_{l}$. We are looking for a matrix $P$ with $N[h]_{m}=P \Psi\left([g]_{m}\right)$. We compute the matrix $P$ by applying the method for computing the representation of elements in $\left(1+\mathfrak{p}^{l}\right) /\left(1+\mathfrak{p}^{m}\right)$ to $N[h]_{m}$. We obtain the representation

$$
\left(\left(\begin{array}{l}
h \\
g
\end{array}\right),\left(\begin{array}{cc}
N & -P \\
0 & M
\end{array}\right)\right)
$$

for $\left(1+\mathfrak{p}^{k}\right) /\left(1+\mathfrak{p}^{m}\right)$. In order to compute $(1+\mathfrak{p}) /\left(1+\mathfrak{p}^{m}\right)$ one computes iteratively

$$
(1+\mathfrak{p}) /\left(1+\mathfrak{p}^{2}\right),(1+\mathfrak{p}) /\left(1+\mathfrak{p}^{4}\right), \ldots,(1+\mathfrak{p}) /\left(1+\mathfrak{p}^{2^{s}}\right),(1+\mathfrak{p}) /\left(1+\mathfrak{p}^{m}\right),
$$

where $s=\left\lfloor\log _{2}(m)\right\rfloor$. 
Algorithm 4.2 (Generators and relations, quadratic method).

Input: $\quad \emptyset_{\mathbf{k}}, \mathfrak{p} \subset \varnothing_{\mathbf{k}}, m \in \mathbb{Z}^{>0}$, integral basis $w$ of $\varnothing_{\mathbf{k}}$

Output: Generators and relations of $(g, M)$ of $(1+\mathfrak{p}) /\left(1+\mathfrak{p}^{m}\right)$

- Compute generators and relations $(h, N)$ of $(1+\mathfrak{p}) /\left(1+\mathfrak{p}^{2}\right)$ :

- Compute matrix representations $A, B \in \mathbb{Z}^{n \times n}$ of $\mathfrak{p}, \mathfrak{p}^{2}$.

○ Set $g \leftarrow\left(g_{1}, \ldots, g_{n}\right)$ with $g_{i}=1+A w_{i}$ for $1 \leq i \leq n$.

- Set $M \leftarrow B A^{-1}$.

○ Set $k \leftarrow 2, s \leftarrow 2$.

$\circ$ While $l \neq m$ :

○ Set $l \leftarrow \min \left(2^{s}, m\right)$.

- Compute generators and relations $(h, N)$ of $\left(1+\mathfrak{p}^{k}\right) /\left(1+\mathfrak{p}^{l}\right)$ :

○ Compute matrix representations $A, B \in \mathbb{Z}^{n \times n}$ of $\mathfrak{p}^{k}, \mathfrak{p}^{l}$.

○ Set $h \leftarrow\left(h_{1}, \ldots, h_{n}\right)$ with $h_{i}=1+A w_{i}$ for $1 \leq i \leq n$.

- Set $N \leftarrow B A^{-1}$.

- Compute $P$, such that $N h=P g$ using Algorithm 4.3 below.

○ Replace $g \leftarrow\left(\begin{array}{c}h \\ g\end{array}\right), M \leftarrow\left(\begin{array}{cc}N & -P \\ 0 & M\end{array}\right)$.

○ Set $k \leftarrow l, s \leftarrow s+1$.

○ Return $(g, M)$.

A representation of the class of $\eta \in \emptyset_{\mathbf{k}}$ in $(1+\mathfrak{p}) /\left(1+\mathfrak{p}^{m}\right)$ by generators as computed with Algorithm 4.2 can be obtained with the following algorithm.

Algorithm 4.3 (Discrete logarithm in $(1+\mathfrak{p}) /\left(1+\mathfrak{p}^{m}\right)$, quadratic method). Input: $\quad$ A maximal order $\emptyset_{\mathbf{k}}$, a integral basis $w=\left(w_{1}, \ldots, w_{n}\right)$ of $\emptyset_{\mathbf{k}}$, a prime ideal $\mathfrak{p} \subset \varnothing_{\mathbf{k}}, m \in \mathbb{N}, \eta \in 1+\mathfrak{p}$

Output: $a$ such that $[a g]=[\eta]$, where $g$ is a set of generators of $(1+\mathfrak{p}) /\left(1+\mathfrak{p}^{m}\right)$ as given by Algorithm 4.2

○ Set $k \leftarrow 1, a \leftarrow()$.

$\circ$ While $k \neq m$ :

○ Set $l \leftarrow \min (2 k, m)$.

- Discrete logarithm in in $\left(1+\mathfrak{p}^{k}\right) /\left(1+\mathfrak{p}^{l}\right)$ :

$\circ$ Find $b \in \mathbb{Z}^{n}$, such that $\eta-1=b w$.

○ Let $A \in \mathbb{Z}^{n \times n}$ be a matrix representation of $\mathfrak{p}^{k}$. Set $c \leftarrow b A^{-1}$.

○ Set $\eta \leftarrow \eta \cdot\left(\prod_{i=1}^{n}\left(1+A w_{i}\right)^{c_{i}}\right)^{-1}$.

- Replace $a \leftarrow(a c), k \leftarrow l$.

$\circ$ Return $a$.

Matrix representations of $\mathfrak{p}, \mathfrak{p}^{2}, \ldots, \mathfrak{p}^{m}$ and the inverses of these matrices can be computed in $O\left(\log m\left(n \mathrm{M}_{\mathfrak{p} m}+\mathrm{T}_{\mathbb{Z}}(n)\right)\right)$ bit operations. Algorithm 4.3 computes a representation of an element of $(1+\mathfrak{p}) /\left(1+\mathfrak{p}^{m}\right)$ in $O\left(n m \log p \mathrm{M}_{\mathfrak{p}^{m}}+\log m \mathrm{D}_{\mathfrak{p}^{m}}\right)$ bit operations. Algorithm 4.2 gives generators and relations of $(1+\mathfrak{p}) /\left(1+\mathfrak{p}^{m}\right)$ in $O\left(n^{2} m \log p \mathrm{M}_{\mathfrak{p}^{m}}+n \log m \mathrm{D}_{\mathfrak{p}^{m}}+\log m \mathbf{T}_{\mathbb{Z}}(n)\right)$ bit operations including the computation of the data above.

Proposition 4.4. A basis of the group $(1+\mathfrak{p}) /\left(1+\mathfrak{p}^{m}\right)$ can be obtained in

$\left.O\left(n m \log p(n+\log m) \mathrm{M}_{\mathfrak{p} m}+n \log m \mathrm{D}_{\mathfrak{p} m}+\log m \mathrm{~T}_{\mathbb{Z}}(n)\right)+\mathrm{S}(n \log m, n \log m)\right)$

bit operations, and the discrete logarithm can be computed in

$$
O\left(n m \log p \mathrm{M}_{\mathfrak{p}^{m}}+\log m \mathrm{D}_{\mathfrak{p}^{m}}+\mathrm{M}(1, n \log m, n \log m)\right) .
$$




\section{5. $\mathfrak{p}$-ADIC LOGARITHMS}

In this section we present a third approach to the computation of the discrete logarithm in $(1+\mathfrak{p}) /\left(1+\mathfrak{p}^{m}\right)$. These methods can also be used for the computation of generators and relations of $(1+\mathfrak{p}) /\left(1+\mathfrak{p}^{m}\right)$ Coh00 chapter 4].

For levels greater than $e /(p-1)$ the $\mathfrak{p}$-adic logarithm can be used for the computation of the discrete logarithm. Define

$$
\log _{\mathfrak{p}}(1+x):=\sum_{i=1}^{\infty}(-1)^{i-1} \frac{x^{i}}{i} \text { and } \exp _{\mathfrak{p}}(x):=\sum_{i=0}^{\infty} \frac{x^{i}}{i !} .
$$

Proposition 5.1. Let $\mathfrak{p}$ be a prime ideal over the prime number $p$ with ramification index $e=v_{\mathfrak{p}}(p)$.

i) The expansion for $\log _{\mathfrak{p}}(1+x)$ converges $\mathfrak{p}$-adically if and only if $v_{\mathfrak{p}}(x) \geq 1$.

ii) Let $x, y \in \mathbf{k}$ with $v_{\mathfrak{p}}(x) \geq 1$ and $v_{\mathfrak{p}}(y) \geq 1$. Then

$$
\log _{\mathfrak{p}}((1+x)(1+y))=\log _{\mathfrak{p}}(1+x) \log _{\mathfrak{p}}(1+y) .
$$

iii) Let $x \in \mathbf{k}$ with $v_{\mathfrak{p}}(x)>e /(p-1)$. Then

$$
\log _{\mathfrak{p}}\left(\exp _{\mathfrak{p}}(x)\right)=x \text { and } \exp _{\mathfrak{p}}\left(\log _{\mathfrak{p}}(1+x)\right)=1+x \text {. }
$$

iv) For any integers $l$ and $m$ with $m>l \geq 1+\lfloor e /(p-1)\rfloor$ the functions $\log _{\mathfrak{p}}$ and $\exp _{\mathfrak{p}}$ are inverse isomorphisms between the multiplicative group $\left(1+\mathfrak{p}^{l}\right) /\left(1+\mathfrak{p}^{m}\right)$ and the additive group $\mathfrak{p}^{l} / \mathfrak{p}^{m}$.

Remark 5.2. Assume $v_{\mathfrak{p}}(x)>e /(p-1)$ and $i=p^{s}$. Then

$$
v_{\mathfrak{p}}\left(x^{i} / i\right)=v_{\mathfrak{p}}\left(x^{p^{s}} / p^{s}\right)=p^{s} v_{\mathfrak{p}}(x)-e s>p^{s} e /(p-1)-e s>e\left(p^{s-1}-s\right) .
$$

The inequality $e\left(p^{s-1}-s\right)>m$ holds if $i=p^{s}>p m \geq p m / e$. Thus $\log _{\mathfrak{p}}$ can be approximated by computing the first $m$ summands of the series for $p \nmid i$ and the summands up to $i=p m$ for $p \mid i$.

The $\mathfrak{p}$-adic logarithm modulo $\mathfrak{p}^{m}$ can be computed in $O\left(m \mathrm{M}_{\mathfrak{p}^{m}}\right)$ bit operations.

Artin-Hasse logarithms yield an inductive method for the computation of the group $(1+p) /\left(1+p^{m}\right)$ of the discrete logarithm similar to the quadratic methods in the previous section. The quadratic methods exploited the isomorphisms $\left(1+p^{k}\right) /\left(1+p^{2 k}\right) \cong \mathfrak{p}^{k} / \mathfrak{p}^{2 k}$; the Artin-Hasse logarithm yields the isomorphisms $\left(1+p^{k}\right) /\left(1+p^{p k}\right) \cong \mathfrak{p}^{k} / \mathfrak{p}^{p k}$. Hence, fewer iterations are necessary using the ArtinHasse logarithm. Define

$$
\mathrm{L}(1+x):=\sum_{i=1}^{p-1}(-1)^{i-1} \frac{x^{i}}{i} \text { and } \mathrm{E}(x):=\sum_{i=0}^{p-1} \frac{x^{i}}{i !} .
$$

Proposition 5.3. Let $\mathfrak{p}$ be a prime ideal over the prime number $p$.

i) The nonzero monomials of the polynomial

$$
\mathrm{L}((1+x)(1+y))-\mathrm{L}(1+x)-\mathrm{L}(1+y)
$$

are of the form $x^{k} y^{l}$ with $k+l \geq p$.

ii) If $v_{\mathfrak{p}}(x)=k$, then $\mathrm{E}(\mathrm{L}(1+x)) \equiv 1+x \bmod \mathfrak{p}^{p k}$.

iii) If $k<l \leq p k$, then the map

$$
\left(1+\mathfrak{p}^{k}\right) /\left(1+p^{l}\right) \rightarrow \mathfrak{p}^{k} / \mathfrak{p}^{l},(1+x) \mapsto \mathrm{L}(1+x),
$$

is a group isomorphism. 


\section{Computing the group of PRincipal units} AND THE DISCRETE LOGARITHM

A major advantage of the basis (respectively unique) representation of the principal units as given in Section 3 is that the structure of the group is given directly. In Section 8 we will see how the fact that we know which basis elements are generators for which level can be exploited in the computation of conductors of ray class groups and more general ideal class groups. Very few computations are needed for the computation of a basis of the multiplicative group of the residue class ring.

We combine the methods from Sections 3, 4, and 5 into a more efficient algorithm. We use the generators given by Theorems 3.3 and 3.6 . the quadratic methods for the discrete logarithm for levels up to $\frac{p e}{p-1}$; for levels greater than $\frac{p e}{p-1}$ we use the $p$-adic logarithm for the computation of the discrete logarithm.

The following algorithm is formulated for case I only. A version for case II requires only minor changes.

Algorithm 6.1 (Discrete logarithm, combined).

Input: $\quad \eta \in 1+\mathfrak{p}, \varnothing_{\mathbf{k}}, \mathfrak{p} \subset \varnothing_{\mathbf{k}}, e, f, p, m \in \mathbb{Z}^{>0}$, a basis $\left(\eta_{11}, \ldots, \eta_{f e}\right)$ of $(1+\mathfrak{p}) /\left(1+\mathfrak{p}^{m}\right)$ as in Theorem [3.3.

Output: $a \in \mathbb{Z}^{e f}$ such that $\left[a^{T}\left(\eta_{11}, \ldots, \eta_{f e}\right)\right]=[\eta]$.

○ Set $k \leftarrow 1, a=\left(a_{11}, \ldots, a_{f e}\right) \leftarrow 0$.

○ While $k<\min \left\{m,\left\lceil\frac{p e}{p-1}\right\rceil\right\}$ :

○ Set $l \leftarrow \min \left\{2 k, m,\left\lceil\frac{p e}{p-1}\right\rceil\right\}$.

○ Compute a basis $\rho_{11}=\eta_{1 t_{1}}^{p^{s_{1}}}, \ldots, \rho_{f 1}=\eta_{f t_{1}}^{p^{s_{1}}}, \ldots, \rho_{f r}=\eta_{f t_{r}}^{p^{s_{t}}}$ of $\left(1+\mathfrak{p}^{k}\right) /\left(1+\mathfrak{p}^{l}\right)$.

$\circ$ Find $\left(c_{11}, \ldots, c_{f r}\right) \in \mathbb{N}$ with $c_{11}\left(\rho_{11}-1\right)+\cdots+c_{f r}\left(\rho_{f r}-1\right)=\eta-1$.

○ Replace $\eta \leftarrow \eta / \prod_{\nu=1}^{r} \prod_{i=1}^{f} \rho_{i \nu}^{c_{i} \nu}$.

○ Replace $a_{1 t_{1}} \leftarrow a_{1 t_{1}}+p^{s_{1}} c_{11}, \ldots, a_{f t_{r}} \leftarrow a_{f t_{r}}+p^{s_{r}} c_{r f}$.

○ Replace $k \leftarrow l$.

○ If $k<m$, then:

$\circ$ Compute a basis $\rho_{11}=\log _{\mathfrak{p}} \eta_{11}^{p^{s_{1}}}, \ldots, \rho_{f 1}=\log _{\mathfrak{p}} \eta_{f 1}^{p^{s_{1}}}, \ldots, \rho_{f e}=\log _{\mathfrak{p}} \eta_{f e}^{p^{s_{e}}}$ of $\mathfrak{p}^{k} / \mathfrak{p}^{m}$.

$\circ$ Set $\gamma \leftarrow \log _{\mathfrak{p}} \eta$. Find $\left(c_{11}, \ldots, c_{f e}\right) \in \mathbb{N}$ with $c_{11} \rho_{11}+\cdots+c_{f e} \rho_{f e}=\gamma$.

○ Replace $a \leftarrow\left(a_{11}+p^{s_{1}} c_{11}, \ldots, a_{e f}+p^{s_{e}} c_{f e}\right)$.

○ Return $a$.

The number of basis elements for $\left(1+\mathfrak{p}^{k}\right) /\left(1+\mathfrak{p}^{l}\right)$ which are $p$-th powers of generators of lower levels is at most $f \frac{p e}{p-1}-e f$. Thus the $\rho_{i \nu}$ for levels less than $\frac{p e}{p-1}$ can be computed in $O\left(\log \frac{p e}{p-1}\left(\frac{p e}{p-1}-e\right) \mathrm{M}_{\mathfrak{p}^{m}}\right)$ bit operations. The $\rho_{i \nu}$ for levels greater than $\frac{p e}{p-1}$ can be computed in $O\left(e f \log p \mathrm{M}_{\mathfrak{p}^{m}}+m \mathrm{M}_{\mathfrak{p}^{m}}\right)$ bit operations. In addition, it is convenient to have matrix representations of the ideal $\mathfrak{p}^{p}, \mathfrak{p}^{p^{2}}, \ldots, \mathfrak{p}^{\frac{p e}{p-1}}, \mathfrak{p}^{m}$ in order to reduce the representations in $\mathfrak{p}^{k} / \mathfrak{p}^{l}$. The matrix representations of these ideals can be computed in $O\left(\log m\left(n \mathrm{M}_{\mathfrak{p}^{m}}+\mathrm{T}_{\mathbb{Z}}(n)\right)\right)$ bit operations. Computing the Hermite normal form of the matrices $\left(\rho_{11} \ldots \rho_{f e}\right)$ in advance will speed up the computation of the discrete logarithm; this can be done in $O\left(\log \frac{p e}{p-1} \mathrm{~T}_{\mathbb{Z}}(n)\right)$. 
Proposition 6.2. In case I and case IIa (case II) a basis (respectively unique) representation of an element in $(1+\mathfrak{p}) /\left(1+\mathfrak{p}^{m}\right)$ can be computed in

$$
O\left(\left(m+\log \frac{p e}{p-1} \text { ef } \log p\right) \mathrm{M}_{\mathfrak{p}^{m}}\right)
$$

bit operations. Assume $m \geq \frac{p e}{p-1}$. Then in case I and case IIa a basis of $(1+\mathfrak{p}) /\left(1+\mathfrak{p}^{m}\right)$ can be computed in

$$
O\left((n \log m+m+e f \log p) \mathrm{M}_{\mathfrak{p}^{m}}+\log m \mathrm{~T}(n)\right)
$$

bit operations. In case II a basis of $(1+\mathfrak{p}) /\left(1+\mathfrak{p}^{m}\right)$ can be computed with the number of bit operations being

$$
O\left(\left(n \log m+m+e f \log p \log \frac{p e}{p-1}\right) \mathrm{M}_{\mathfrak{p} m}+\log m \mathbf{T}(n)+\mathrm{S}(e f)\right) .
$$

The quadratic method from Section 4 is implemented in the computer algebra system PARI/GP [BB $\left.{ }^{+} 99\right]$. In the computer algebra systems KASH/KANT $\mathrm{Po}^{+} 00$ DF ${ }^{+}$96] and MAGMA [BC95] a combination of the methods from Sections 3 and 4 is used.

\section{COMPuting RAY Class GROUPS}

Let $\mathfrak{m}_{0}$ be an integral ideal and $\mathfrak{m}_{\infty}$ a formal product of real infinite places of $\mathbf{k}$. Then $\mathfrak{m}:=\mathfrak{m}_{0} \mathfrak{m}_{\infty}$ is called a congruence module. Let $a^{(i)} \in \mathbb{C}$ be the $i$ th conjugate of $a \in \mathbf{k}$. Multiplicative congruences with respect to a finite place contained in the congruence module are defined by

$$
a \equiv 1 \bmod ^{*} \mathfrak{p}^{m}: \Leftrightarrow v_{\mathfrak{p}}(a-1) \geq m
$$

and for a real infinite place $\mathfrak{p}_{\infty}^{(i)}$ by

$$
a \equiv 1 \bmod ^{*}\left(\mathfrak{p}_{\infty}^{(i)}\right)^{m}: \Leftrightarrow a^{(i)}>0 .
$$

Let $\mathbf{I}^{\mathfrak{m}}:=\left\{\mathfrak{a} \in \mathbf{I}_{\mathbf{k}} \mid \operatorname{gcd}\left(\mathfrak{a}, \mathfrak{m}_{0}\right)=1\right\}$ and $\mathbf{H}_{\mathfrak{m}}:=\left\{(a) \in \mathbf{H}_{\mathbf{k}} \mid a \equiv 1 \bmod ^{*} \mathfrak{m}\right\}$. Then the ray class group modulo $\mathfrak{m}$ is defined as $\mathbf{C l}_{\mathbf{k}}^{\mathfrak{m}}:=\mathbf{I}^{\mathfrak{m}} / \mathbf{H}_{\mathfrak{m}}$.

Let $\mathbf{H}^{\mathfrak{m}}:=\left\{\mathfrak{a} \in \mathbf{H}_{\mathbf{k}} \mid \operatorname{gcd}\left(\mathfrak{a}, \mathfrak{m}_{0}\right)=1\right\}, \mathbf{k}_{\mathfrak{m}}:=\left\{a \in \mathbf{k} \mid a \equiv 1 \bmod ^{*} \mathfrak{m}\right\}$, $\mathbf{k}^{\mathfrak{m}}:=\left\{a \in \mathbf{k} \mid \operatorname{gcd}\left((a), \mathfrak{m}_{0}\right)=1\right\}, \mathbf{U}_{\mathbf{k}}$ the group of units of $\varnothing_{\mathbf{k}}$ and $\mathbf{U}_{\mathfrak{m}}:=\{u \in$ $\left.\mathbf{U}_{\mathbf{k}} \mid u \equiv 1 \bmod ^{*} \mathfrak{m}\right\}$. We will compute the ray class group $\mathbf{C l}_{\mathbf{k}}^{\mathfrak{m}}$ in the way the following diagram from Lan94 suggests:

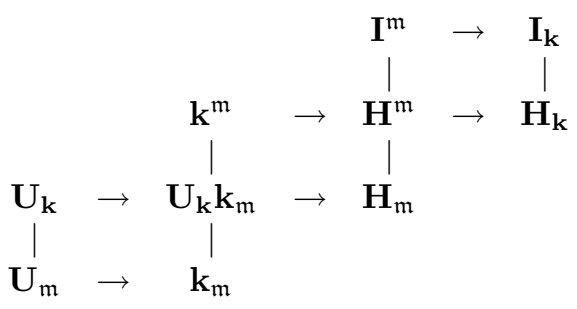

We note that two opposite vertical lines represent isomorphisms of factor groups. Horizontal arrows denote natural embeddings.

Algorithm 7.1 (Ray class group). Input: $\quad \emptyset_{\mathbf{k}}, \mathbf{U}_{\mathbf{k}}, \mathbf{C l}_{\mathbf{k}}, \mathfrak{m}=\mathfrak{m}_{0} \mathfrak{m}_{\infty}$. Output: $\mathbf{C l}_{\mathbf{k}}^{\mathfrak{m}}$.

$\circ$ Compute $\mathbf{k}^{\mathfrak{m}} / \mathbf{k}_{\mathfrak{m}} \cong\left(\varnothing_{\mathbf{k}} / \mathfrak{m}_{0}\right)^{*} \times \prod_{\mathfrak{p} \mid \mathfrak{m}_{\infty}} \mathbb{R}^{*} / \mathbb{R}^{*>0}$.

- Compute $\mathbf{U}_{\mathbf{k}} / \mathbf{U}_{\mathfrak{m}}$ via the image of $\mathbf{U}_{\mathbf{k}}$ in $\mathbf{k}^{\mathfrak{m}} / \mathbf{k}_{\mathfrak{m}}$.

$\circ$ Factor $\left(\mathbf{k}^{\mathfrak{m}} / \mathbf{k}_{\mathfrak{m}}\right)$ by $\left(\mathbf{U}_{\mathbf{k}} / \mathbf{U}_{\mathfrak{m}}\right)$. 
- Compute generators of $\mathbf{C l}_{\mathbf{k}}$ which are prime to $\mathfrak{m}_{0}$.

- Compute a basis of $\mathbf{C l}_{\mathbf{k}}^{\mathfrak{m}}$.

We already described the computation of $\left(\varnothing_{\mathbf{k}} / \mathfrak{m}_{0}\right)^{*}$. For the computation of $\mathbf{k}^{\mathfrak{m}} / \mathbf{k}_{\mathfrak{m}}$ we also need generators of $\mathbf{k}^{\mathfrak{m} \infty} / \mathbf{k}_{\mathfrak{m}_{\infty}} \cong \prod_{\mathfrak{p} \mid \mathfrak{m}_{\infty}} \mathbb{R}^{*} / \mathbb{R}^{*>0}$. Assume $\mathfrak{m}_{\infty}=$ $\mathfrak{p}_{\infty}^{\left(\nu_{1}\right)} \cdots \mathfrak{p}_{\infty}^{\left(\nu_{s}\right)}$. We are looking for generators $\vartheta_{i}$ of the group $\mathbf{k}^{\mathfrak{m}_{\infty}} / \mathbf{k}_{\mathfrak{m}_{\infty}}$ satisfying $\vartheta_{i} \equiv 1 \bmod ^{*} \mathfrak{m}_{0}$. The approximation theorem assures the existence of $\vartheta_{1}, \ldots, \vartheta_{s}$ subject to

$$
\begin{aligned}
& \vartheta_{j} \equiv-1 \quad \bmod ^{*} \mathfrak{p}_{\infty}^{\left(\nu_{j}\right)}, \\
& \vartheta_{j} \equiv 1 \bmod ^{*} \mathfrak{p}_{\infty}^{\left(\nu_{i}\right)}, \quad i=1, \ldots, s, \quad i \neq j, \\
& \vartheta_{j} \equiv 1 \bmod ^{*} \mathfrak{m}_{0} \text {. }
\end{aligned}
$$

Let $a_{1}, \ldots, a_{n}$ be a basis of $\mathfrak{m}_{0}$, and let $\mathbf{x} \in \mathbb{Z}^{n}$ be a solution of the system of inequalities

$$
\begin{aligned}
& 1+x_{1} a_{1}^{\left(\nu_{j}\right)}+\cdots+x_{n} a_{n}^{\left(\nu_{j}\right)}<0, \\
& 1+x_{1} a_{1}^{\left(\nu_{i}\right)}+\cdots+x_{n} a_{n}^{\left(\nu_{i}\right)}>0, \quad i=1, \ldots, s, \quad i \neq j .
\end{aligned}
$$

Then $\vartheta_{j}=\mathbf{x}\left(a_{1}, \ldots, a_{n}\right)^{\mathrm{T}}$ satisfies the congruences listed above. To obtain $\mathbf{x}$ we solve a system of linear equations

$$
\begin{aligned}
1+\bar{x}_{1} a_{1}^{\left(\nu_{j}\right)}+\cdots+\bar{x}_{n} a_{n}^{\left(\nu_{j}\right)} & =-a, \\
1+\bar{x}_{1} a_{1}^{\left(\nu_{i}\right)}+\cdots+\bar{x}_{n} a_{n}^{\left(\nu_{i}\right)} & =\quad a, \quad i=1, \ldots, s, i \neq j,
\end{aligned}
$$

for $a:=\frac{1}{2} \sum_{i=1}^{n}\left|a_{i}\right|$. From the real solution $\overline{\mathbf{x}}$ of that system we then obtain $\mathbf{x}$ by rounding all coordinates to their closest integer: $x_{i}=\left\lfloor\bar{x}_{i}\right\rceil \quad(1 \leq i \leq s)$.

We compute the group $\mathbf{U} / \mathbf{U}_{\mathfrak{m}}$ via the image of $\mathbf{U}$ in $\mathbf{k}^{\mathfrak{m}} / \mathbf{k}_{\mathfrak{m}}$. For this we calculate the images of a system of generators of $\mathbf{U}$ in $\mathbf{k}^{\mathfrak{m}} / \mathbf{k}_{\mathfrak{m}}$. Then the factor group of the finite groups $\mathbf{k}^{\mathfrak{m}} / \mathbf{k}_{\mathfrak{m}}$ and $\mathbf{U} / \mathbf{U}_{\mathfrak{m}}$ is calculated by computing a normal form for the matrix of relations for the generators as outlined in CDO96.

Algorithms for the computation of class groups of algebraic number fields are presented in [Coh93], Hes96], and [PZ89]. Generators of the class group which are prime to $\mathfrak{m}_{0}$ are easily calculated by algorithm 7 in Coh96. It remains to compute the factor group of $\mathbf{I}^{\mathfrak{m}} / \mathbf{H}^{\mathfrak{m}}$ and $\mathbf{H}^{\mathfrak{m}} / \mathbf{H}_{\mathfrak{m}}$ to get $\mathbf{C l}_{\mathbf{k}}^{\mathfrak{m}}=\mathbf{I}^{\mathfrak{m}} / \mathbf{H}_{\mathfrak{m}}$.

\section{Computing CONDuCtors, Discriminants, And Signatures}

Let $\mathfrak{m}$ be a congruence module and $\mathbf{C l}_{\mathbf{k}}^{\mathfrak{m}}=\mathbf{I}^{\mathfrak{m}} / \mathbf{H}_{\mathfrak{m}}$ be the ray class group modulo $\mathfrak{m}$. Let $\mathbf{J}_{\mathfrak{m}}$ be an ideal group which can be defined with $\mathfrak{m}$, i.e., which satisfies $\mathbf{I}^{\mathfrak{m}} \supset \mathbf{J}_{\mathfrak{m}} \supset \mathbf{H}_{\mathfrak{m}}$

The conductor $\mathfrak{f}$ of an ideal group is the smallest congruence module $\mathfrak{f}$ with which that ideal group can be defined.

In the sequel we explain how to calculate conductors of ray class groups. It is clear from the diagram of the previous section that we do not need to compute the entire ray class group. It clearly suffices to compute $\mathbf{H}^{\mathfrak{m}} / \mathbf{H}_{\mathfrak{m}} \cong\left(\mathbf{k}^{\mathfrak{m}} / \mathbf{k}_{\mathfrak{m}}\right) /\left(\mathbf{U} / \mathbf{U}_{\mathfrak{m}}\right)$. An ideal group which can be defined with $\mathfrak{m}_{1}$ and with $\mathfrak{m}_{2}$ can also be defined with $\operatorname{gcd}\left(\mathfrak{m}_{1}, \mathfrak{m}_{2}\right)$. Hence, if for each $\mathfrak{p} \mid \mathfrak{m}$ the integer $j_{\mathfrak{p}}$ is the largest exponent such that $\mathfrak{n}_{\mathfrak{p}}=\mathfrak{m} / \mathfrak{p}^{j_{\mathfrak{p}}}$ is integral and $\mathbf{H}^{\mathfrak{n}_{\mathfrak{p}}} / \mathbf{H}_{\mathfrak{n}_{\mathfrak{p}}}=\mathbf{H}^{\mathfrak{m}} / \mathbf{H}_{\mathfrak{m}}$, then $\mathfrak{f}$ is is the greatest common divisor of all these $\mathfrak{n}_{\mathfrak{p}}$. We determine $j_{\mathfrak{p}}$ as the largest integer $i$ for which the groups $\mathbf{H}^{\mathfrak{m}} / \mathbf{H}_{\mathfrak{m}}$ and $\mathbf{H}^{\mathfrak{m} / \mathfrak{p}^{i}} / \mathbf{H}_{\mathfrak{m} / \mathfrak{p}^{i}}$ still coincide. 
It can be easily deduced from Theorems 3.3 and 3.6 that for any prime ideal $\mathfrak{p}$ and any $k>0$ the same representatives for the classes of generators can be used for $\mathbf{k}^{\mathfrak{p}^{k}} / \mathbf{k}_{\mathfrak{p}^{k}}$ and $\mathbf{k}^{\mathfrak{p}^{k-1}} / \mathbf{k}_{\mathfrak{p}^{k-1}}$. With respect to basis representations of $\left(\varnothing_{\mathbf{k}} / \mathfrak{p}^{k}\right)^{*}$ we only need to change the orders of the generators to get a basis for $\left(\varnothing_{\mathbf{k}} / \mathfrak{p}^{k-1}\right)^{*}$. In the case of a unique representation, also the relation for $\eta_{1 e_{0}}^{p^{\mu_{0}}}$ must be replaced by the order of $\eta_{1 e_{0}}$ in the step from level $k=\frac{p e}{p-1}$ to level $k-1=\frac{p e}{p-1}-1$.

Since the same representatives for the classes of generators of $\mathbf{k}^{\mathfrak{p}^{k}} / \mathbf{k}_{\mathfrak{p}^{k}}$ and $\mathbf{k}^{\mathfrak{p}^{k-1}} / \mathbf{k}_{\mathfrak{p}^{k-1}}$ can be chosen, the representation of the image of the generators of $\mathbf{U}$ by the generators of $\mathbf{k}^{\mathfrak{p}^{k}} / \mathbf{k}_{\mathfrak{p}^{k}}$ is a representation by the generators of $\mathbf{k}^{\mathfrak{p}^{k-1}} / \mathbf{k}_{\mathfrak{p}^{k-1}}$ as well. So the relation matrix of $\left(\mathbf{k}^{\mathfrak{p}^{k-1}} / \mathbf{k}_{\mathfrak{p}^{k-1}}\right) /\left(\mathbf{U} / \mathbf{U}_{\mathfrak{p}^{k-1}}\right)$ can be derived from the relation matrix of $\left(\mathbf{k}^{\mathfrak{p}^{k}} / \mathbf{k}_{\mathfrak{p}^{k}}\right) /\left(\mathbf{U} / \mathbf{U}_{\mathfrak{p}^{k}}\right)$ by a simple change of the orders of the generators and, in the case of a unique representation, also by replacing the relation for $\eta_{1 e_{0}}$ as described above.

The same can be done for $\left(\mathbf{k}^{\mathfrak{n}} / \mathbf{k}_{\mathfrak{n}}\right) /\left(\mathbf{U} / \mathbf{U}_{\mathfrak{n}}\right)$ and $\left(\mathbf{k}^{\mathfrak{n} / \mathfrak{p}} / \mathbf{k}_{\mathfrak{n} / \mathfrak{p}}\right) /\left(\mathbf{U} / \mathbf{U}_{\mathfrak{n} / \mathfrak{p}}\right)$, since they are direct products of groups $\left(\varnothing_{\mathbf{k}} / \mathfrak{p}^{k}\right)^{*}$ and the corresponding groups for the infinite places. A comparison can easily be carried out via the Hermite normal forms of the corresponding relation matrices. It is even faster to consider the diagonal elements of the Hermite normal form of the first relation matrix and to determine whether the changed orders for the second matrix change the Hermite normal form. The infinite places are treated similarly.

Let $\mathbf{J}_{\mathfrak{m}}$ be an ideal group defined with $\mathfrak{m}$. For $\mathfrak{n} \mid \mathfrak{m}$ let $\Phi$ denote the surjection from $\mathbf{C l}_{\mathbf{k}}^{\mathfrak{m}}$ to $\mathbf{C l}_{\mathbf{k}}^{\mathfrak{n}}$. For the computation of the conductor of $\mathbf{I}^{\mathfrak{m}} / \mathbf{J}_{\mathfrak{m}}$ we use the fact that $\mathbf{C l}_{\mathbf{k}}^{\mathfrak{m}} /\left(\mathbf{J}_{\mathfrak{m}} / \mathbf{H}_{\mathfrak{m}}\right) \cong \mathbf{I}^{\mathfrak{m}} / \mathbf{J}_{\mathfrak{m}}$. For $\mathbf{C l}_{\mathbf{k}}^{\mathfrak{n}} / \Phi\left(\mathbf{J}_{\mathfrak{m}} / \mathbf{H}_{\mathfrak{m}}\right)=\mathbf{C l}_{\mathbf{k}}^{\mathfrak{m}} /\left(\mathbf{J}_{\mathfrak{m}} / \mathbf{H}_{\mathfrak{m}}\right)$ the ideal class group $\mathbf{I}^{\mathfrak{m}} / \mathbf{J}_{\mathfrak{m}}$ can also be defined with $\mathfrak{n}$. We proceed as in the case of ray class groups; we compare the groups $\mathbf{C l}_{\mathbf{k}}^{\mathfrak{n}} / \Phi\left(\mathbf{J}_{\mathfrak{m}} / \mathbf{H}_{\mathfrak{m}}\right)$ and $\mathbf{C l}_{\mathbf{k}}^{\mathfrak{m}} /\left(\mathbf{J}_{\mathfrak{m}} / \mathbf{H}_{\mathfrak{m}}\right)$ via their relation matrices, which in addition to the relations for $\mathbf{H}^{\mathfrak{m}} / \mathbf{H}_{\mathfrak{m}}$ contain the relations for the generators of $\mathbf{C l}_{\mathbf{k}}$ and the relation for the factorisation by $\mathbf{J}_{\mathfrak{m}} / \mathbf{H}_{\mathfrak{m}}$.

Let $\mathbf{K} / \mathbf{k}$ be the ray class field corresponding to the module $\mathfrak{m}$. In CDO98. Theorem 3.3] Cohen, Oliver and Diaz y Diaz develop a formula for the relative discriminant $\mathfrak{d}_{\mathbf{K} / \mathbf{k}}$. For $\mathfrak{n} \mid \mathfrak{m}$ set $h_{\mathfrak{n}, \mathbf{J}_{\mathfrak{m}}}:=\#\left(\mathbf{C l}_{\mathbf{k}}^{\mathfrak{n}} / \Phi\left(\mathbf{J}_{\mathfrak{m}} / \mathbf{H}_{\mathfrak{m}}\right)\right)$. Then the relative discriminant is

$$
\mathfrak{d}_{\mathbf{K} / \mathbf{k}}=\prod_{\mathfrak{p} \mid \mathfrak{m}_{0}} \mathfrak{p}^{m_{\mathfrak{p}} h_{\mathfrak{m}, J_{\mathfrak{m}}}-\sum_{1 \leq k \leq \mathfrak{m}_{\mathfrak{p}}} h_{\mathfrak{m} / \mathfrak{p} k, J_{\mathfrak{m}}}} .
$$

The ray class numbers $h_{\mathfrak{m} / \mathfrak{p}^{k}, \mathbf{J}_{\mathfrak{m}}}$ can be easily computed with the same methods which we used for the conductor. Finally, let $\left(r_{1}, r_{2}\right)$ be the signature of $\mathbf{k}$ and $\left(R_{1}, R_{2}\right)$ the signature of $\mathbf{K}$. Specialising the formula for signatures of ray class fields from [CDO98, Theorem 3.3], we get $R_{1}=r_{1} h_{\mathfrak{f}, \mathbf{J}_{\mathfrak{m}}}$.

\section{The Global FUnCtion Field CASE}

In this section we discuss the theory and algorithms in the global function field case.

9.1. Ray divisor class groups. Let $\mathbf{k} / \mathbb{F}_{q}$ denote a global function field over the finite field of $q$ elements and characteristic $p$. Unlike the number field case, for global function fields there is no canonical maximal order. It is hence more natural to work with divisors instead of ideals. Let $\mathbf{D}$ denote the group of divisors and $\mathbf{P}$ 
the subgroup of principal divisors $(a), a \in \mathbf{k}^{*}$. The divisor class group $\mathbf{C l}$ is defined as $\mathbf{D} / \mathbf{P}$.

Let $\mathfrak{m} \in \mathbf{D}$ be an effective divisor, which we again call a congruence module. For $a \in \mathbf{k}$ we say that $a \equiv 1 \bmod ^{*} \mathfrak{m}$, if $v_{\mathfrak{p}}(a-1) \geq v_{\mathfrak{p}}(\mathfrak{m})$ for all places $\mathfrak{p} \mid \mathfrak{m}$. We further define the subgroups $\mathbf{D}^{\mathfrak{m}}:=\{\mathfrak{a} \in \mathbf{D} \mid \operatorname{gcd}(\mathfrak{a}, \mathfrak{m})=0\}, \mathbf{P}^{\mathfrak{m}}:=\mathbf{P} \cap \mathbf{D}^{\mathfrak{m}}, \mathbf{k}^{\mathfrak{m}}:=\{a \in$ $\left.\mathbf{k}^{*} \mid(a) \in \mathbf{P}^{\mathfrak{m}}\right\}, \mathbf{k}_{\mathfrak{m}}:=\left\{a \in \mathbf{k}^{\mathfrak{m}} \mid a \equiv 1 \bmod ^{*} \mathfrak{m}\right\}$ and $\mathbf{P}_{\mathfrak{m}}:=\left\{(a) \in \mathbf{P}^{\mathfrak{m}} \mid a \in \mathbf{k}_{\mathfrak{m}}\right\}$. The ray divisor class group $\mathbf{C l}_{\mathfrak{m}}$ for the congruence module $\mathfrak{m}$ is defined as $\mathbf{D}^{\mathfrak{m}} / \mathbf{P}_{\mathfrak{m}}$.

Let $H$ be a subgroup of $\mathbf{D}^{\mathfrak{m}}$, and let $U_{H}:=\left\{a \in \mathbf{k}^{\mathfrak{m}} \mid(a) \in H\right\}$. We then obtain the following diagram, similar to the number field case:

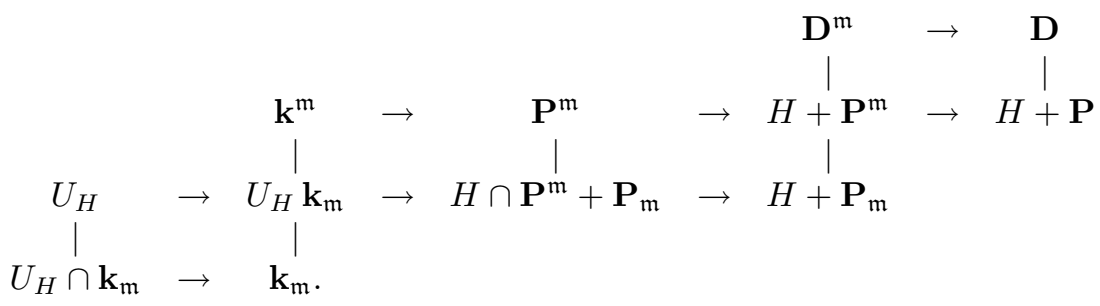

Again, vertical lines refer to isomorphisms of factor groups and horizontal arrows denote natural embeddings. For $H=\{0\}$ we have $U_{H}=\mathbb{F}_{q}^{*}$. Using this in the diagram above, we get the canonical exact sequence of abelian groups

$$
0 \longrightarrow \mathbb{F}_{q}^{*} \longrightarrow \mathbf{k}^{\mathfrak{m}} / \mathbf{k}_{\mathfrak{m}} \longrightarrow \mathbf{C l}_{\mathfrak{m}} \longrightarrow \mathbf{D}^{\mathfrak{m}} / \mathbf{P}^{\mathfrak{m}} \longrightarrow 0 .
$$

In view of [CDO98, Proposition 1.2], this means that in order to compute $\mathbf{C l}_{\mathfrak{m}}$ we need to compute generators and relations for $\mathbf{D}^{\mathfrak{m}} / \mathbf{P}^{\mathfrak{m}}$ and $\mathbf{k}^{\mathfrak{m}} / \mathbb{F}_{q}^{*} \mathbf{k}_{\mathfrak{m}}$. To link these we also need to compute the actual elements of $\mathbf{k}^{\mathfrak{m}} / \mathbb{F}_{q}^{*} \mathbf{k}_{\mathfrak{m}}$ corresponding to relations of $\mathbf{D}^{\mathfrak{m}} / \mathbf{P}_{\mathfrak{m}}$ and to express these in the generators of $\mathbf{k}^{\mathfrak{m}} / \mathbb{F}_{q}^{*} \mathbf{k}_{\mathfrak{m}}$.

The computation of generators and relations for $\mathbf{C l}=\mathbf{D} / \mathbf{P}$ is described in [Hes99. From these, generators and relations corresponding to divisors and principal divisors in $\mathbf{D}^{\mathfrak{m}}$ and $\mathbf{P}^{\mathfrak{m}}$ respectively can for example be obtained by applying the approximation theorem analogously to the number field case. The actual element in $\mathbf{k}^{\mathfrak{m}}\left(\bmod \mathbb{F}_{q}^{*} \mathbf{k}_{\mathfrak{m}}\right)$ corresponding to a relation, i.e., the generator of a principal divisor in $\mathbf{P}^{\mathfrak{m}}$, can be computed efficiently. Furthermore, it is also possible to find the expression of a divisor class (given by a divisor) in the generators of $\mathbf{C l}$. For details we refer to [Hes99.

Once generators and relations for $\mathbf{C l}_{\mathfrak{m}}$ are computed as above. The task of expressing a given element of $\mathbf{C l}_{\mathfrak{m}}$ represented by a divisor $\mathfrak{a} \in \mathbf{D}^{\mathfrak{m}}$ can be solved as follows: We first express $\mathfrak{a}$ in the representatives of the generators of $\mathbf{D}^{\mathfrak{m}} / \mathbf{P}^{\mathfrak{m}}$, up to a principal divisor $(b)$ in $\mathbf{P}^{\mathfrak{m}}$ for $b \in \mathbf{k}^{\mathfrak{m}}$. Then $b$ is expressed in the representatives of the generators of $\mathbf{k}^{\mathfrak{m}} / \mathbb{F}_{q}^{*} \mathbf{k}_{\mathfrak{m}}$, up to an element of $\mathbb{F}_{q}^{*} \mathbf{k}_{\mathfrak{m}}$.

9.2. Multiplicative groups of residue class rings. We are left to describe how generators and relations for $\mathbf{k}^{\mathfrak{m}} / \mathbb{F}_{q}^{*} \mathbf{k}_{\mathfrak{m}}$ are computed and how an element of $\mathbf{k}^{\mathfrak{m}}$ $\left(\bmod \mathbb{F}_{q}^{*} \mathbf{k}_{\mathfrak{m}}\right)$ can be expressed in these generators.

We first consider $\mathbf{k}^{\mathfrak{m}} / \mathbf{k}_{\mathfrak{m}}$. Let $\varnothing_{\mathfrak{p}}$ denote the valuation ring of $\mathfrak{p}$ such that $\mathfrak{p}$ is the maximal ideal of $\varnothing_{\mathfrak{p}}$. It is straightforward to see that the canonical map of multiplicative groups $\mathbf{k}^{\mathfrak{m}} \longrightarrow \prod_{\mathfrak{p} \mid \mathfrak{m}}\left(\varnothing_{\mathfrak{p}} / \mathfrak{p}^{v_{\mathfrak{p}}(\mathfrak{m})}\right)^{*}$ has kernel $\mathbf{k}_{\mathfrak{m}}$. Using the weak approximation theorem, we also see that it is surjective, so that we obtain $\mathbf{k}^{\mathfrak{m}} / \mathbf{k}_{\mathfrak{m}}$ $\cong \prod_{\mathfrak{p} \mid \mathfrak{m}}\left(\varnothing_{\mathfrak{p}} / \mathfrak{p}^{v_{\mathfrak{p}}(\mathfrak{m})}\right)^{*}$, in analogy to the number field case. Once we have generators and relations for each of the $\left(\varnothing_{\mathfrak{p}} / \mathfrak{p}^{v_{\mathfrak{p}}(\mathfrak{m})}\right)^{*}$, their disjoint union will give generators 
and relations for $\mathbf{k}^{\mathfrak{m}} / \mathbf{k}_{\mathfrak{m}}$. We remark that we do not have to compute actual elements of $\mathbf{k}^{\mathfrak{m}}$. We obtain generators and relations for $\mathbf{k}^{\mathfrak{m}} / \mathbb{F}_{q}^{*} \mathbf{k}_{\mathfrak{m}}$ by expressing a generator of $\mathbb{F}_{q}^{*}$ in the generators of $\mathbf{k}^{\mathfrak{m}} / \mathbf{k}_{\mathfrak{m}}$ and by adding the obtained expression to the relations of $\mathbf{k}^{\mathfrak{m}} / \mathbf{k}_{\mathfrak{m}}$. Finally, to find the expression of an element of $\mathbf{k}^{\mathfrak{m}}$ $\left(\bmod \mathbb{F}_{q}^{*} \mathbf{k}_{\mathfrak{m}}\right)$ in the generators, we do so for every local factor $\left(\varnothing_{\mathfrak{p}} / \mathfrak{p}^{v_{\mathfrak{p}}(\mathfrak{m})}\right)^{*}$.

We next need to explain how generators and relations for $\left(\varnothing_{\mathfrak{p}} / \mathfrak{p}^{m}\right)^{*}$ for $m \in \mathbb{Z} \geq 1$ can be determined and how an element in $\varnothing_{\mathfrak{p}}^{*}\left(\bmod \mathfrak{p}^{m}\right)$ can be expressed in these generators. In analogy to the number field case we have

$$
\left(\varnothing_{\mathfrak{p}} / \mathfrak{p}^{m}\right)^{*} \cong\left(\emptyset_{\mathfrak{p}} / \mathfrak{p}\right)^{*} \times(1+\mathfrak{p}) /\left(1+\mathfrak{p}^{m}\right),
$$

where $\left(\varnothing_{\mathfrak{p}} / \mathfrak{p}\right)^{*}$ is mapped to the group of $\left(q^{\operatorname{deg}(\mathfrak{p})}-1\right)$-th roots of unity in $\left(\varnothing_{\mathfrak{p}} / \mathfrak{p}^{m}\right)^{*}$.

It is well known that the order of $(1+\mathfrak{p}) /\left(1+\mathfrak{p}^{m}\right)$ is $\left(q^{\operatorname{deg}(\mathfrak{p})}\right)^{m-1}$. For $a \in \mathbb{R}^{\geq 0}$ and setting $\min \{\}:=0$, define $\lceil a\rceil_{p}:=\min \left\{p^{l} \mid a \leq p^{l}, l \in \mathbb{Z}^{\geq 0}\right\}$. The following lemma immediately gives us generators and relations for $(1+\mathfrak{p}) /\left(1+\mathfrak{p}^{m}\right)$.

Lemma 9.1. Let $\pi \in \mathfrak{p} \backslash \mathfrak{p}^{2}$, and let $B$ be a system of representatives in $\emptyset_{\mathfrak{p}}$ of a basis of the $\mathbb{F}_{p}$-vector space $\varnothing_{\mathfrak{p}} / \mathfrak{p}$. Let $U_{\beta, j}$ denote the subgroup of $(1+\mathfrak{p}) /\left(1+\mathfrak{p}^{m}\right)$ generated by $\left(1+\beta \pi^{j}\right) U_{\mathfrak{p}}^{(m)}$ for $\beta \in B$ and $j \geq 1, j$ coprime to $p$. The groups $U_{\beta, j}$ have pairwise trivial intersection and are of order $\left|U_{\beta, j}\right|=\lceil m / j\rceil_{p}$. Moreover,

$$
(1+\mathfrak{p}) /\left(1+\mathfrak{p}^{m}\right)=\prod\left\{U_{\beta, j} \mid \beta \in B, 1 \leq j \leq m-1, \operatorname{gcd}(j, p)=1\right\} .
$$

Proof. This follows from [Aue99, p. 39].

Assume that for given $a \in(1+\mathfrak{p}) /\left(1+\mathfrak{p}^{m}\right)$ we want to express $a$ as a power product of the generators of the lemma. The most elementary way of achieving this is by a Gaussian elimination procedure as done in Algorithm 3.5. The methods of Section 4 can also be used. The $\mathfrak{p}$-adic logarithm in Section 5 does not carry over, because we work in characteristic $p$ and would have to divide by $p$ in the series expansion of $\log _{\mathfrak{p}}$ and $\exp _{\mathfrak{p}}$. However, the Artin-Hasse logarithm can be applied; Proposition 5.3 remains true in characteristic $p$. This appears to be a very efficient way for computing the expression of $a$ in the generators.

9.3. Conductors. Let $H$ be a subgroup of $\mathbf{C l}_{\mathfrak{m}}$. If $\mathfrak{m}^{\prime} \leq \mathfrak{m}$ is another congruence module, we have a natural epimorphism $\Phi_{\mathfrak{m}^{\prime}}: \mathbf{C l}_{\mathfrak{m}} \longrightarrow \mathbf{C l}_{\mathfrak{m}^{\prime}}$. There is a unique smallest congruence module $\mathfrak{f} \leq \mathfrak{m}$ such that $\mathbf{C l}_{\mathfrak{f}} / \Phi_{\mathfrak{f}}(H) \cong \mathbf{C l}_{\mathfrak{m}} / H$. This congruence module is called the conductor of $H$. The conductor of $\mathbf{C l}_{\mathfrak{m}}$ is defined to be the conductor of its zero subgroup $H=\{0\}$.

Using the definition, there is a straightforward way of computing the conductor of a subgroup $H$ of $\mathbf{C l}_{\mathfrak{m}}$. Namely, we successively check for all smaller congruence modules $\mathfrak{m}^{\prime}<\mathfrak{m}$ whether the isomorphy holds. If not, we take the next $\mathfrak{m}^{\prime}$. If yes, we replace $\mathfrak{m}$ by $\mathfrak{m}^{\prime}$ and $H$ by $\Phi(H)$ and start from the beginning. At the end, $\mathfrak{m}$ is smallest possible. Because of the uniqueness property, $\mathfrak{m}$ must be the conductor of the original $H$.

A more explicit way is given by the diagram above. Assume the subgroup of $\mathbf{C l}_{\mathfrak{m}}$ is the image of a divisor group $H \subseteq \mathbf{D}^{\mathfrak{m}}$. The conductor is a smallest congruence module $\mathfrak{m}^{\prime}$ such that $U_{H} \mathbf{k}_{\mathfrak{m}^{\prime}} / \mathbf{k}_{\mathfrak{m}^{\prime}} \cong U_{H} \mathbf{k}_{\mathfrak{m}} / \mathbf{k}_{\mathfrak{m}}$. In the case of $H=\{0\}$ we see from the exact sequence above, and since the cardinality of $\left(\varnothing_{\mathfrak{p}} / \mathfrak{p}^{m}\right)^{*}$ changes if $m$ changes, that $\mathfrak{m}$ already is the conductor of $\mathbf{C l}_{\mathfrak{m}}$. The computation of the conductor for general $H$ using the isomorphy amounts to two major steps. 
In the first step we need to determine generators of $U_{H}$. If $h_{1}, \ldots, h_{r}$ are the divisor classes in $\mathbf{C l}$ of the generators of $H$, we compute the kernel of $\mathbb{Z}^{r} \longrightarrow \mathbf{C l}$, $\left(\lambda_{i}\right)_{i} \mapsto \sum_{i} \lambda_{i} h_{i}$. To carry out this computation we need to know generators and relations of $\mathbf{C l}$ and need to express the classes $h_{i}$ in these generators. From the kernel we obtain a basis of $H \cap \mathbf{P}^{\mathfrak{m}}$ and can reconstruct the corresponding elements in $U_{H}$.

In the second step we express the generators of $U_{H}$ in the generators of $\mathbf{k}^{\mathfrak{m}} / \mathbf{k}_{\mathfrak{m}}$. Joining these expressions and the relations of $\mathbf{k}^{\mathfrak{m}} / \mathbf{k}_{\mathfrak{m}}$ yields the relations of $\mathbf{k}^{\mathfrak{m}} / U_{H} \mathbf{k}_{\mathfrak{m}}$. Now, analogously to the number field case, for smaller $\mathfrak{m}^{\prime}$ there is no need to recompute the expressions for the generators of $U_{H}$. We only have to adjust the orders of the generators of $\mathbf{k}^{\mathfrak{m}} / \mathbf{k}_{\mathfrak{m}}$ in the relation matrix for $\mathbf{k}^{\mathfrak{m}^{\prime}} / U_{H} \mathbf{k}_{\mathfrak{m}^{\prime}}$. Once we obtain a different relation module, $\mathfrak{m}^{\prime}$ is too small. Successive testing and descending again exhibits the smallest congruence module preserving the isomorphy.

9.4. Class field discriminants and genera. Let $\mathfrak{m}$ be a congruence module. From class field theory we know that the finite abelian extensions $\mathbf{K} / \mathbf{k}$ of conductor less than or equal to $\mathfrak{m}$ correspond inclusion-reversing to subgroups $H$ of $\mathbf{C l}_{\mathfrak{m}}$ of finite index. The conductor of $\mathbf{K} / \mathbf{k}$ is equal to the conductor of $H$.

Let $h_{\mathfrak{m}^{\prime}, H}:=\mathbf{C l}_{\mathfrak{m}^{\prime}} / \Phi_{\mathfrak{m}^{\prime}}(H)$ for the natural epimorphism $\Phi_{\mathfrak{m}^{\prime}}: \mathbf{C l}_{\mathfrak{m}} \longrightarrow \mathbf{C l}_{\mathfrak{m}^{\prime}}$, and let $\mathfrak{d}_{\mathbf{K} / \mathbf{k}}$ denote the discriminant of $\mathbf{K} / \mathbf{k}$. Using the same proof as in CDO98, we obtain

$$
\mathfrak{d}_{\mathbf{K} / \mathbf{k}}=\left(h_{\mathfrak{m}, H}\right) \mathfrak{m}-\sum_{\mathfrak{p} \mid \mathfrak{m}}\left(\sum_{k=1}^{v_{\mathfrak{p}}(\mathfrak{m})} h_{\mathfrak{m}-k \mathfrak{p}, H}\right) \mathfrak{p} .
$$

Let $\operatorname{deg}(H):=\min \left\{\operatorname{deg}(\mathfrak{a}) \mid \mathfrak{a}+\mathbf{P}_{\mathfrak{m}} \in H\right\}$. Because $H$ is of finite index in $\mathbf{C l}_{\mathfrak{m}}$, we have $\operatorname{deg}(H) \geq 1$. Let $\mathbb{F}_{q_{1}}$ be the full constant field of $\mathbf{K}$. From class field theory we have $[\mathbf{K}: \mathbf{k}]=h_{\mathfrak{m}, H}$ and $\left[\mathbb{F}_{q_{1}}: \mathbb{F}_{q}\right]=\operatorname{deg}(H)$. Furthermore, the norm of the different of $\mathbf{K} / \mathbf{k}$ down to $\mathbf{k}$ equals $\mathfrak{d}_{\mathbf{K} / \mathbf{k}}$, and $\left[\mathbb{F}_{q_{1}}: \mathbb{F}_{q}\right]$ times the degree of the different equals the degree of the discriminant. If $g_{\mathbf{k}}$ and $g_{\mathbf{K}}$ denote the genus of $\mathbf{k}$ and $\mathbf{K}$ respectively we obtain from the Hurwitz genus formula

$$
\operatorname{deg}(H)\left(g_{\mathbf{K}}-1\right)=h_{\mathfrak{m}, H}\left(g_{\mathbf{k}}-1+\frac{\operatorname{deg}(\mathfrak{m})}{2}\right)-\frac{1}{2} \sum_{\mathfrak{p} \mid \mathfrak{m}}\left(\sum_{k=1}^{v_{\mathfrak{p}}(\mathfrak{m})} h_{\mathfrak{m}-k \mathfrak{p}, H}\right) \operatorname{deg}(\mathfrak{p}) .
$$

Using the methods from Section 9.3 we can compute the numbers $h_{\mathfrak{m}-k \mathfrak{p}, H}$ easily. We are thus able to determine the discriminant $\mathfrak{d}_{\mathbf{K} / \mathbf{k}}$ and the genus $g_{\mathbf{K}}$.

\section{REFERENCES}

[Aue99] Roland Auer, Ray Class Fields of Global Function Fields with Many Rational Places, Carl-von-Ossietzky-Universität, Oldenburg, 1999 (see also Acta Arith. 95 (2000), 97122). MR 2002e: 11162

$\left[\mathrm{BB}^{+} 99\right]$ C. Batut, K. Belabas, D. Bernardi, H. Cohen, M. Olivier, The Computer Algebra System PARI-GP, Université Bordeaux I, 1999, ftp://megrez . math.u-bordeaux.fr/pub/pari/

[BC95] W. Bosma and J.J. Cannon, Handbook of Magma functions, School of Mathematics, University of Sydney, Sydney, 1995.

[CDO96] Henri Cohen, Francisco Diaz y Diaz, and Michel Olivier, Computing ray class groups, conductors and discriminants, ANTS II (Henri Cohen, ed.), LNCS 1122, Springer, 1996, 49-57. MR 98c: 11123

[CDO98] Henri Cohen, Francisco Diaz y Diaz, and Michel Olivier, Computing ray class groups, conductors and discriminants, Math. Comp. 67 (1998). MR 98g:11128 
[Coh93] Henri Cohen, A course in computational algebraic number theory, Springer Verlag, New York, 1993. MR 94i:11105

[Coh96] Henri Cohen, Hermite and Smith normal form algorithms over Dedekind domains, Math. Comp. 65 (1996), 1681-1699. MR 97e:11159

[Coh00] Henri Cohen, Advanced topics in computational number theory, Springer Verlag, New York, 2000. MR 2000k:11144

$\left[\mathrm{DF}^{+}\right.$96] M. Daberkow, C. Fieker, J. Klüners, M. Pohst, K. Roegner, M. Schörnig, and K. Wildanger, KANT V4, J. Symb. Comp. 11 (1996), 267-283. MR 99g:11150

[Fie00] Claus Fieker, Computing class fields via the Artin map, Math. Comp. 70 (2001), no. 235, 1293-1303. MR 2002e:11153

[Has80] Helmut Hasse, Number theory, Springer Verlag, Berlin, 1980. MR 81c:12001b

[Hes96] Florian Heß, Zur Klassengruppenberechnung in algebraischen Zahlkörpern, Diplomarbeit, TU - Berlin, 1996, http://www.math.TU-Berlin.DE/〜kant/publications/diplom/hess.ps.gz.

[Hes99] Florian Heß, Zur Divisorenklassengruppenberechnung in globalen Funktionenkörpern, PhD thesis, TU - Berlin, 1999, http://www.math.TU-Berlin.DE/ kant/publications/diss/diss_FH.ps.gz.

[Lan94] Serge Lang, Algebraic number theory, 2nd ed., Graduate Texts in Mathematics, vol. 110, Springer Verlag, Berlin, 1994. MR 95f:11085

[Pau96] Sebastian Pauli, Zur Berechnung von Strahlklassengruppen, Diplomarbeit, TU - Berlin, 1996, http://www.math.TU-Berlin.DE/ kant/publications/diplom/pauli.ps.gz.

[PZ89] Michael E. Pohst and Hans Zassenhaus, Algorithmic algebraic number theory, Cambridge University Press, 1989. MR 92b:11074

$\left[\mathrm{Po}^{+} 00\right]$ Michael E. Pohst et al, The computer algebra system KASH/KANT, TU-Berlin, 2000, http://www.math.tu-berlin.de/ ${ }^{\text {kant/. }}$

[SWD] Oliver Schirokauer, Damian Weber, and Thomas Denny, Discrete Logarithms: The Effectiveness of the Index Calculus Method, ANTS II (Henri Cohen, ed.), LNCS 1122, Springer, 1996, 337-361. MR 98i:11109

[Sim94] Charles C. Sims, Computation with finitely presented groups, Cambridge University Press, 1994. MR 95f:20053

Institut für Mathematik, MA 8-1, Technische Universität Berlin, Strasse des 17. Juni 136, 10623 Berlin, Germany

Current address: Department of Computer Science, University of Bristol, BS8 1UB, England

E-mail address: florian@cs.bris.ac.uk

Institut für Mathematik, MA 8-1, Technische Universität Berlin, Strasse des 17. Juni 136, 10623 Berlin, Germany

E-mail address: pauli@math.tu-berlin.de

Institut für Mathematik, MA 8-1, Technische Universität Berlin, Strasse des 17. Juni 136, 10623 Berlin, Germany

E-mail address: pohst@math.tu-berlin.de 